\title{
Generalized ASOR and Modified ASOR Methods for Saddle Point Problems
}

\author{
Zhengge Huang, Ligong Wang, Zhong Xu, and Jingjing Cui \\ Department of Applied Mathematics, Northwestern Polytechnical University, Xian, Shaanxi 710072, China \\ Correspondence should be addressed to Zhengge Huang; zhenggehuang@mail.nwpu.edu.cn
}

Received 19 December 2015; Revised 15 March 2016; Accepted 27 March 2016

Academic Editor: Ruben Specogna

Copyright (C) 2016 Zhengge Huang et al. This is an open access article distributed under the Creative Commons Attribution License, which permits unrestricted use, distribution, and reproduction in any medium, provided the original work is properly cited.

Recently, the accelerated successive overrelaxation- (SOR-) like (ASOR) method was proposed for saddle point problems. In this paper, we establish a generalized accelerated SOR-like (GASOR) method and a modified accelerated SOR-like (MASOR) method, which are extension of the ASOR method, for solving both nonsingular and singular saddle point problems. The sufficient conditions of the convergence (semiconvergence) for solving nonsingular (singular) saddle point problems are derived. Finally, numerical examples are carried out, which show that the GASOR and MASOR methods have faster convergence rates than the SORlike, generalized SOR (GSOR), modified SOR-like (MSOR-like), modified symmetric SOR (MSSOR), generalized symmetric SOR (GSSOR), generalized modified symmetric SOR (GMSSOR), and ASOR methods with optimal or experimentally found optimal parameters when the iteration parameters are suitably chosen.

\section{Introduction}

Consider the following large and sparse saddle point problem:

$$
\mathscr{A} u=\left(\begin{array}{cc}
A & B \\
-B^{T} & 0
\end{array}\right)\left(\begin{array}{l}
x \\
y
\end{array}\right)=\left(\begin{array}{c}
p \\
-q
\end{array}\right)
$$

where $A \in \mathbb{R}^{m \times m}$ is a symmetric positive definite matrix, $B \in$ $\mathbb{R}^{m \times n}, p \in \mathbb{R}^{m}$, and $q \in \mathbb{R}^{n}(m \geq n)$. It follows that the linear system (1) is nonsingular when $B$ is of full column rank and singular when $B$ is rank deficient [1].

The saddle point problem (1) is important and arises in a variety of scientific and engineering applications, such as mixed finite element approximation of elliptic partial differential equations, optimal control, computational fluid dynamics, weighted least-squares problems, electronic networks, and computer graphics; see [1-4] and references therein.

When the linear system (1) is nonsingular, a number of iterative methods and their numerical properties have been discussed to approximate the unique solution of nonsingular saddle point problem (1) in the literatures, such as SORlike methods [5-11], Uzawa-type methods [5, 12-18], Hermitian and skew-Hermitian splitting (HSS) methods and their variants [2,19-24], restrictively preconditioned conjugate gradient (RPCG) methods [25, 26], and preconditioned Krylov subspace iteration methods [27-29].

In the case of $B$ in (1) being rank deficient, we call the linear system (1) a singular saddle point problem. For this case, various kinds of relaxation iteration methods have also been established. In [30-34], the authors applied the inexact Uzawa methods for singular saddle point problems.

Recently, Njeru and Guo [35] developed an accelerated SOR-like (ASOR) method for the nonsingular saddle point problem (1) and numerical experiments results show that the convergence rate of the ASOR method is faster than the convergence rates of the SOR-like, GSSOR, and GSOR methods when the parameters are suitably chosen. It can be described as follows.

For the coefficient matrix of the saddle point problem (1), Njeru and Guo [35] made the following splitting:

$$
\mathscr{A}=D-L-U,
$$


where

$$
\begin{aligned}
& D=\left(\begin{array}{cc}
\alpha A & 0 \\
0 & Q
\end{array}\right), \\
& L=\left(\begin{array}{cc}
-A & 0 \\
B^{T} & \frac{1}{2} Q
\end{array}\right), \\
& U=\left(\begin{array}{cc}
\alpha A & -B \\
0 & \frac{1}{2} Q
\end{array}\right),
\end{aligned}
$$

with $\alpha>0, \omega \neq 0$, and $Q \in \mathbb{R}^{n \times n}$ is a symmetric positive definite matrix. The iteration for ASOR method [35] is thus described by the algorithm below.

The ASOR Method. Given initial vectors $x^{(0)} \in \mathbb{R}^{m}$ and $y^{(0)} \in$ $\mathbb{R}^{n}$ and two real relaxation factors $\alpha>0$ and $\omega \neq 0$, for $k=0,1,2, \ldots$, until the iteration sequence $\left\{\left(x^{(k)^{T}}\right),\left(y^{(k)^{T}}\right)\right\}$ converges to the exact solution of the saddle point problem (1), compute

$$
\begin{aligned}
& x^{(k+1)}=\frac{\alpha}{\alpha+\omega} x^{(k)}-\frac{\omega}{\alpha+\omega} A^{-1}\left(B y^{k}-p\right), \\
& y^{(k+1)}=y^{(k)}+\frac{2 \omega}{2-\omega} Q^{-1}\left(B^{T} x^{(k+1)}-q\right) .
\end{aligned}
$$

In addition, the authors in [35] obtained the experimentally found optimal parameters by using trial and error method in different cases, and the experimentally found optimal values for $\alpha$ and $\omega$ are very close to

$$
\begin{gathered}
\alpha=\frac{\sqrt{\mu_{\max } \mu_{\min }}}{8 \sqrt{\mu_{\min }}+\sqrt{\mu_{\max }}}, \\
\omega=\frac{2 \sqrt{\mu_{\min } \mu_{\max }}+1}{\left(\sqrt{\mu_{\min }}+\sqrt{\mu_{\max }}\right)^{2}},
\end{gathered}
$$

where $\mu_{\max }$ and $\mu_{\min }$ are the maximum and minimum eigenvalues of the matrix $Q^{-1} B^{T} A^{-1} B$, respectively.

In this paper, based on the ASOR method, by adding the new parameters, the generalized ASOR (GASOR) method and the modified ASOR (MASOR) method are proposed to solve the nonsingular and the singular saddle point problem (1). We discuss the convergence properties of the GASOR and MASOR methods for solving nonsingular saddle point problems and the semiconvergence properties of the GASOR and MASOR methods for solving singular saddle point problems, respectively. In addition, the choice of the relaxation parameters of the GASOR and MASOR methods is discussed in Section 4. Numerical examples are implemented to illustrate the effectiveness of the GASOR and MASOR methods with appropriate parameters for both nonsingular and singular saddle point problems.

The rest of this paper is organized as follows. In Section 2, we propose the GASOR method for solving nonsingular and singular saddle point problem (1) and discuss the convergence (semiconvergence) properties of the GASOR method for solving nonsingular (singular) saddle point problems. The MASOR method is proposed and convergence (semiconvergence) properties of the MASOR method for solving nonsingular (singular) saddle point problems are derived in Section 3. The analysis of the optimal convergence factors of two new methods is presented in Section 4. In Section 5, numerical experiments are provided to examine the feasibility and effectiveness of the GASOR and MASOR methods for solving both nonsingular and singular saddle point problems. Finally, some conclusions are drawn.

\section{The Generalized ASOR Method for Saddle Point Problems}

2.1. The Generalized ASOR Method. In this section, we propose the generalized ASOR (GASOR) method for solving the saddle point problem (1). The GASOR method with appropriate parameters has the faster convergence rate than those of SOR-like [8], GSOR [5], MSOR-like [9], and ASOR [35] methods with optimal or experimentally found optimal parameters for nonsingular saddle point problems. For the coefficient matrix of the augmented system (1), we make the splitting of $\mathscr{A}$ as in (2).

Let $\omega$ and $\tau$ be two nonzero reals, let $I_{m} \in \mathbb{R}^{m \times m}$ and $I_{n} \in \mathbb{R}^{n \times n}$ be the $m$-by- $m$ and the $n$-by- $n$ identity matrices, respectively, and

$$
\Omega=\left(\begin{array}{cc}
\omega I_{m} & 0 \\
0 & \tau I_{n}
\end{array}\right)
$$

Then, we consider the following generalized ASOR iteration scheme for solving the saddle point problem (1):

$$
\left(\begin{array}{c}
x^{(k+1)} \\
y^{(k+1)}
\end{array}\right)=M_{\alpha, \omega, \tau}\left(\begin{array}{c}
x^{(k)} \\
y^{(k)}
\end{array}\right)+(D-\Omega L)^{-1} \Omega\left(\begin{array}{c}
p \\
-q
\end{array}\right),
$$

where $M_{\alpha, \omega, \tau}$ is the iteration matrix of the GASOR method and its form as follows:

$$
\begin{aligned}
& M_{\alpha, \omega, \tau}=(D-\Omega L)^{-1}[(I-\Omega) D+\Omega U] \\
& =\left(\begin{array}{cc}
(\alpha+\omega) A & 0 \\
-\tau B^{T} & \frac{2-\tau}{2} Q
\end{array}\right)^{-1}\left(\begin{array}{cc}
\alpha A & -\omega B \\
0 & \frac{2-\tau}{2} Q
\end{array}\right) \\
& =\left(\begin{array}{cc}
\frac{1}{\alpha+\omega} A^{-1} & 0 \\
\frac{2 \tau}{(2-\tau)(\alpha+\omega)} Q^{-1} B^{T} A^{-1} & \frac{2}{2-\tau} Q^{-1}
\end{array}\right)\left(\begin{array}{cc}
\alpha A & -\omega B \\
0 & \frac{2-\tau}{2} Q
\end{array}\right) \\
& =\left(\begin{array}{cc}
\frac{\alpha}{\alpha+\omega} I_{m} & -\frac{\omega}{\alpha+\omega} A^{-1} B \\
\frac{2 \tau \alpha}{(2-\tau)(\alpha+\omega)} Q^{-1} B^{T} & I_{n}-\frac{2 \tau \omega}{(2-\tau)(\alpha+\omega)} Q^{-1} B^{T} A^{-1} B
\end{array}\right) .
\end{aligned}
$$

Then, we have the following algorithmic description of the GASOR method.

The GASOR Method. Given initial vectors $x^{(0)} \in \mathbb{R}^{m}$ and $y^{(0)} \in \mathbb{R}^{n}$ and three real relaxation factors $\alpha>0$ and $\omega, \tau \neq 0$, 
for $k=0,1,2, \ldots$, until the iteration sequence $\left\{\left(x^{(k)^{T}}\right),\left(y^{(k)^{T}}\right)\right\}$ converges to the exact solution of the saddle point problem (1), compute

$$
\begin{aligned}
& x^{(k+1)}=\frac{\alpha}{\alpha+\omega} x^{(k)}-\frac{\omega}{\alpha+\omega} A^{-1}\left(B y^{k}-p\right), \\
& y^{(k+1)}=y^{(k)}+\frac{2 \tau}{2-\tau} Q^{-1}\left(B^{T} x^{(k+1)}-q\right) .
\end{aligned}
$$

Note that the GASOR method involves three parameters $\alpha, \omega$, and $\tau$. Since

$$
(D-\Omega L)=\left(\begin{array}{cc}
(\alpha+\omega) A & 0 \\
-\tau B^{T} & \frac{2-\tau}{2} Q
\end{array}\right),
$$

then the matrix $(D-\Omega L)$ is invertible if and only if

$$
\begin{array}{r}
\alpha+\omega \neq 0, \\
\tau \neq 2,
\end{array}
$$

by the definiteness of $A$ and $Q$.

Note that the GASOR method with $\tau=\omega$ reduces to the ASOR method proposed by Njeru and Guo [35].

\subsection{Convergence of the GASOR Method}

Lemma 1. Suppose that $\mu$ is an eigenvalue of matrix $Q^{-1} B^{T} A^{-1} B$, if $\lambda$ satisfies

$$
(\lambda-1)(2-\tau)(\alpha-\alpha \lambda-\lambda \omega)=2 \lambda \tau \omega \mu,
$$

and then $\lambda$ is an eigenvalue of iteration matrix $M_{\alpha, \omega, \tau}$. Conversely, if $\lambda$ is an eigenvalue of $M_{\alpha, \omega, \tau}$ such that $\lambda \neq 1, \tau \neq 2$, $\lambda \neq \alpha /(\alpha+\omega)$, and $\mu$ satisfy (12), then $\mu$ is a nonzero eigenvalue of matrix $Q^{-1} B^{T} A^{-1} B$.

Proof. Let $\lambda$ be an eigenvalue of $M_{\alpha, \omega, \tau}$ and let $\left(x^{T}, y^{T}\right)^{T}$ be the corresponding eigenvector. Then we have

$$
M_{\alpha, \omega, \tau}\left(\begin{array}{l}
x \\
y
\end{array}\right)=\lambda\left(\begin{array}{l}
x \\
y
\end{array}\right) .
$$

Equation (13) can be written as

$$
\begin{aligned}
& \left(\begin{array}{cc}
\alpha A & -\omega B \\
0 & \left(1-\frac{\tau}{2}\right) Q
\end{array}\right)\left(\begin{array}{l}
x \\
y
\end{array}\right) \\
& =\lambda\left(\begin{array}{cc}
(\alpha+\omega) A & 0 \\
-\tau B^{T} & \left(1-\frac{\tau}{2}\right) Q
\end{array}\right)\left(\begin{array}{l}
x \\
y
\end{array}\right),
\end{aligned}
$$

which is equivalent to

$$
\begin{aligned}
& (\alpha-\alpha \lambda-\lambda \omega) A x=\omega B y, \\
& \frac{(\lambda-1)(2-\tau)}{2} Q y=\lambda \tau B^{T} x .
\end{aligned}
$$

Inasmuch as $\alpha-\alpha \lambda-\lambda \omega \neq 0$, we obtain

$$
x=\frac{\omega}{\alpha-\alpha \lambda-\lambda \omega} A^{-1} B y
$$

from (15). Substituting (17) into (16), we have

$$
\frac{(\lambda-1)(2-\tau)}{2} y=\frac{\lambda \tau \omega}{\alpha-\alpha \lambda-\lambda \omega} Q^{-1} B^{T} A^{-1} B y
$$

by the definiteness of $Q$.

If $\mu$ satisfies (12), then we have

$$
Q^{-1} B^{T} A^{-1} B y=\mu y .
$$

If $y=0$, then from (17) this leads to $x=0$, which is a contradiction that $\left(x^{T}, y^{T}\right)^{T}$ is an eigenvector. Thus, $y \neq 0$, and therefore $\mu$ is an eigenvalue of $Q^{-1} B^{T} A^{-1} B$. Furthermore, let $\lambda=1$; from (15) and (16), we have $\alpha A x-\omega B y=(\alpha+\omega) A x$ and $\tau B^{T} x=0$. Thus, $x=-A^{-1} B y$ by $\omega \neq 0$ and the definiteness of $A$, which yields that $B^{T} A^{-1} B y=0$. However $A$ is positive definite, so is $A^{-1}$, which implies that $B y=0$ and then $x=-A^{-1} B y=0$ and $y=0$ by $B$ which is full column rank. This contradicts the assumption that $\left(x^{T}, y^{T}\right)^{T}$ is an eigenvector as well. Therefore, combining (12) and the assumptions $\tau \neq 2, \lambda \neq \alpha /(\alpha+\omega)$ results in $\mu \neq 0$; that is, $\mu$ is a nonzero eigenvalue of the matrix $Q^{-1} B^{T} A^{-1} B$.

We can prove the second assertion by reversing the process.

One may easily show that for the special case $\tau=\omega$ the GASOR method reduces to the ASOR method derived in [35].

Lemma 2 (see [36]). Both roots of the real quadratic equation $x^{2}-b x+c=0$ are less than one in modulus if and only if $|c|<1$ and $|b|<1+c$.

Theorem 3. Let $A$ and $Q$ be symmetric positive definite, and let $B$ be full column rank. Assume that all eigenvalues $\mu$ of $Q^{-1} B^{T} A^{-1} B$ are real and positive. Then, the GASOR method is convergent for all $\alpha, \omega$, and $\tau$ such that

$$
\frac{\omega \tau \mu_{\max }}{2-\tau}<2 \alpha+\omega, \quad \omega>0, \alpha>0,0<\tau<2,
$$

where $\mu_{\text {max }}$ is the maximum eigenvalue of $Q^{-1} B^{T} A^{-1} B$.

Proof. After some manipulations on Lemma 1, we have

$$
\begin{gathered}
(\alpha+\omega)(\tau-2) \lambda^{2}+((\omega+2 \alpha)(2-\tau)-2 \tau \omega \mu) \lambda \\
+\alpha(\tau-2)=0 .
\end{gathered}
$$

Changing the above equation into $x^{2}-b x+c=0$, we find

$$
\lambda^{2}+\left(\frac{2 \tau \omega \mu}{(\alpha+\omega)(2-\tau)}-\frac{2 \alpha+\omega}{\alpha+\omega}\right) \lambda+\frac{\alpha}{\alpha+\omega}=0,
$$

where

$$
\begin{aligned}
& b=\frac{2 \alpha+\omega}{\alpha+\omega}-\frac{2 \tau \omega \mu}{(\alpha+\omega)(2-\tau)}, \\
& c=\frac{\alpha}{\alpha+\omega} .
\end{aligned}
$$


By Lemma 2, $|\lambda|<1$ if and only if

$$
\begin{gathered}
\left|\frac{\alpha}{\alpha+\omega}\right|<1, \\
\left|\frac{2 \alpha+\omega}{\alpha+\omega}-\frac{2 \tau \omega \mu}{(\alpha+\omega)(2-\tau)}\right|<\frac{\alpha}{\alpha+\omega}+1 .
\end{gathered}
$$

It follows from (24) that

$$
\begin{array}{r}
\omega>0, \\
-2 \alpha<\omega .
\end{array}
$$

It is obvious that (26) holds true by $\alpha>0$ and $\omega>0$. From (25), we get

$$
0<\frac{\omega \tau \mu}{2-\tau}<2 \alpha+\omega
$$

Since $\omega>0, \tau>0$, and $\mu$ is an real and positive eigenvalue of matrix $Q^{-1} B^{T} A^{-1} B$, we have

$$
\begin{gathered}
0<\tau<2, \\
\frac{\omega \tau \mu}{2-\tau}<2 \alpha+\omega .
\end{gathered}
$$

However,

$$
\frac{\omega \tau \mu}{2-\tau} \leq \frac{\omega \tau \mu_{\max }}{2-\tau}
$$

We obtain that if the conditions (20) are satisfied, the GASOR method is convergent.

From (21), we get the following corollary.

Corollary 4. Let $A$ and $Q$ be symmetric positive definite, let and $B$ be full column rank. If $\mu$ is an eigenvalue of the matrix $Q^{-1} B^{T} A^{-1} B$, then eigenvalues of the matrix $M_{\alpha, \omega, \tau}$ are given by $\lambda=\alpha /(\alpha+\omega)$ or

$$
\begin{aligned}
\lambda & =\frac{1}{2}\left[\left(\frac{2 \alpha+\omega}{\alpha+\omega}-\frac{2 \omega \tau \mu}{(2-\tau)(\alpha+\omega)}\right)\right. \\
& \left. \pm \sqrt{\left(\frac{2 \omega \tau \mu}{(2-\tau)(\alpha+\omega)}-\frac{2 \alpha+\omega}{\alpha+\omega}\right)^{2}-\frac{4 \alpha}{\alpha+\omega}}\right] .
\end{aligned}
$$

2.3. Semiconvergence of the GASOR Method for Singular Saddle Point Problems. For singular saddle point problems, in [37], Wang and Zhang studied the accelerated HSS (AHSS) method first put forward by Bai and Golub in [20] for solving singular saddle point problems; Yang and $\mathrm{Wu}$ [38] proposed the Uzawa-HSS method and applied it for singular saddle point problems [39]. Recently, Miao and Cao [40] studied the semiconvergence of the generalized local HSS method established by Zhu [41] for singular saddle point problems; Zhou and Zhang [42] discussed the semiconvergence of the GMSSOR method which derived by Zhang et al. in [43] for singular saddle point problems. In the sequel, Chen and $\mathrm{Ma}$
[44] presented a generalized shift-splitting preconditioner and investigated this preconditioner for singular saddle point problems [45]. For more literatures on this theme, one can refer to $[46,47]$ and references therein.

In this section, by using the idea of [42], we assume that $B$ in (1) is rank deficient; that is, $\operatorname{rank}(B)<n$; that is, $\mathscr{A}$ in (1) is singular. In addition, we will discuss the semiconvergence region for parameters $\alpha, \omega$, and $\tau$ in the GASOR method for solving the singular saddle point problem (1). The GASOR method has a faster convergence rate than the ones of the GSSOR [48], MSSOR [49], and GMSSOR [42, 43] methods for singular saddle point problems when optimal or experimentally found optimal parameters are chosen for them.

We first recollect some needed known results about stationary iterative methods for singular linear systems. We denote the range and the null space of the matrix $A$ by $R(A)$ and $N(A)$, respectively. The smallest nonnegative integer $i$ such that $\operatorname{rank}\left(A^{i}\right)=\operatorname{rank}\left(A^{i+1}\right)$ is called the index of $A$ and is denoted by $i=\operatorname{index}(A)$. For a matrix $A \in \mathbb{C}^{m \times m}$, the splitting $A=M-N$ is a nonsingular splitting if $M$ is nonsingular. Let $T=M^{-1} N$ and $c=M^{-1} b$, then solving linear systems $A x=b$ is equivalent to considering the following iterative scheme:

$$
x^{(k+1)}=T x^{(k)}+c .
$$

When $A$ is singular, the semiconvergence about the iteration scheme (31) is precisely described in $[50,51]$.

Definition 5 (see [51]). For any initial vector $z^{(0)}$, the iteration scheme (31) is semiconvergence to a solution $z^{*}$ of $A x=$ $b$ if and only if the iteration matrix $T$ is semiconvergent. Moreover, it holds that $z^{*}=(I-T)^{D} c+[I-(I-T)(I-$ $\left.T)^{D}\right] z^{(0)}$, where $I$ is the identity matrix with appropriate size and $(I-T)^{D}$ denotes the Drazin inverse of $I-T$.

Definition 6 (see [42]). The pseudospectral radius of matrix $T$ is defined as

$$
\mathscr{V}(T)=\max \{|\lambda| \mid \lambda \in \sigma(T) \backslash\{1\}\},
$$

where $\sigma(T)$ stands for the spectrum of matrix $T$.

Lemma 7 (see [52]). The iteration (31) is semiconvergent, if and only if the following conditions hold:

(1) $\operatorname{index}(I-T)=1$.

(2) $\mathscr{V}(T)<1$.

Theorem 8. Let $A$ and $Q$ be symmetric positive definite, and let $B$ be rank deficient. If $\mu$ is an eigenvalue of the matrix $Q^{-1} B^{T} A^{-1} B$, then eigenvalues of the matrix $M_{\alpha, \omega, \tau}$ are $1, \lambda=$ $\alpha /(\alpha+\omega)$, and the remainder eigenvalues are the roots of the quadratic equation (21).

Proof. In order to seek for the eigenvalues of $M_{\alpha, \omega, \tau}$, we need to solve $\operatorname{det}\left(\lambda I-M_{\alpha, \omega, \tau}\right)=0$ : 


$$
\left|\lambda I-M_{\alpha, \omega, \tau}\right|=\left|\begin{array}{cc}
\left(\lambda-\frac{\alpha}{\alpha+\omega}\right) I_{m} & \frac{\omega}{\alpha+\omega} A^{-1} B \\
-\frac{2 \tau \alpha}{(2-\tau)(\alpha+\omega)} Q^{-1} B^{T} & (\lambda-1) I_{n}+\frac{2 \tau \omega}{(2-\tau)(\alpha+\omega)} Q^{-1} B^{T} A^{-1} B
\end{array}\right| .
$$

$$
\text { If } \lambda=\alpha /(\alpha+\omega) \text {, we have }
$$

$$
\begin{aligned}
\left|\lambda I-M_{\alpha, \omega, \tau}\right| & =\left|\begin{array}{cc}
0 & \frac{\omega}{\alpha+\omega} A^{-1} B \\
-\frac{2 \tau \alpha}{(2-\tau)(\alpha+\omega)} Q^{-1} B^{T} & -\frac{\omega}{\alpha+\omega} I_{n}+\frac{2 \tau \omega}{(2-\tau)(\alpha+\omega)} Q^{-1} B^{T} A^{-1} B
\end{array}\right| \\
& =\left|\begin{array}{cc}
0 & \frac{\omega}{\alpha+\omega} A^{-1} B \\
-\frac{2 \tau \alpha}{(2-\tau)(\alpha+\omega)} Q^{-1} B^{T} & -\frac{\omega}{\alpha+\omega} I_{n}
\end{array}\right|=\left|\begin{array}{cc}
-\frac{2 \tau \alpha}{(2-\tau)(\alpha+\omega)} A^{-1} B Q^{-1} B^{T} & 0 \\
-\frac{2 \tau \alpha}{(2-\tau)(\alpha+\omega)} Q^{-1} B^{T} & -\frac{\omega}{\alpha+\omega} I_{n}
\end{array}\right| \\
& =\left[-\frac{2 \tau \alpha}{(2-\tau)(\alpha+\omega)}\right]^{m}\left(-\frac{\omega}{\alpha+\omega}\right)^{n}\left|A^{-1} B Q^{-1} B^{T}\right| .
\end{aligned}
$$

Notice that $Q$ is symmetric positive definite and therefore $\operatorname{rank}\left(A^{-1} B Q^{-1} B^{T}\right)=\operatorname{rank}\left(B Q^{-1} B^{T}\right)=\operatorname{rank}\left(B^{T}\right)=$ $\operatorname{rank}(B)<n$. As $A^{-1} B Q^{-1} B^{T}$ is an $m \times m(m \geq n)$ matrix, we have $\left|A^{-1} B Q^{-1} B^{T}\right|=0$, which implies that $\alpha /(\alpha+\omega)$ is an eigenvalue of $M_{\alpha, \omega, \tau}$. If $\lambda \neq \alpha /(\alpha+\omega)$, after some elementary transformation of matrix, the following holds:

$$
\begin{aligned}
\left|\lambda I-M_{\alpha, \omega, \tau}\right| & =\left|\begin{array}{cc}
\left(\lambda-\frac{\alpha}{\alpha+\omega}\right) I_{m} & \frac{\omega}{\alpha+\omega} A^{-1} B \\
0 & (\lambda-1) I_{n}+\frac{2 \tau \omega \lambda}{[(\alpha+\omega) \lambda-\alpha](2-\tau)} Q^{-1} B^{T} A^{-1} B
\end{array}\right| \\
& =\left(\lambda-\frac{\alpha}{\alpha+\omega}\right)^{m}\left|\begin{array}{cc}
I_{m} & \frac{\omega}{\alpha+\omega} A^{-1} B \\
0 & (\lambda-1) I_{n}+\frac{2 \tau \omega \lambda}{[(\alpha+\omega) \lambda-\alpha](2-\tau)} Q^{-1} B^{T} A^{-1} B
\end{array}\right| .
\end{aligned}
$$

Suppose that $\operatorname{rank}(B)=n-k(k>0)$. Notice that $A$ and $Q$ are symmetric and positive definite, so $\operatorname{rank}\left(Q^{-1} B^{T} A^{-1} B\right)=$ $\operatorname{rank}\left(B^{T} A^{-1} B\right)=\operatorname{rank}(B)$. Then, $Q^{-1} B^{T} A^{-1} B$ has at least $k$ eigenvalues 0 , and we denote them by $\mu_{n-k+1}=\mu_{n-k+2}=$ $\cdots=\mu_{n}=0$. Let $V \in \mathbb{R}^{n \times n}$ be an orthogonal matrix such that $V^{T} Q^{-1} B^{T} A^{-1} B V=R$, where $R$ is an upper triangular matrix, whose diagonal elements are composed of the eigenvalues of $Q^{-1} B^{T} A^{-1} B$. Then, we have

$$
\left|\begin{array}{cc}
I_{m} & \frac{\omega}{\alpha+\omega} A^{-1} B \\
0 & (\lambda-1) I_{n}+\frac{2 \tau \omega \lambda}{[(\alpha+\omega) \lambda-\alpha](2-\tau)} Q^{-1} B^{T} A^{-1} B
\end{array}\right|
$$$$
=\left|(\lambda-1) I_{n}+\frac{2 \tau \omega \lambda}{[(\alpha+\omega) \lambda-\alpha](2-\tau)} Q^{-1} B^{T} A^{-1} B\right|
$$$$
=\left|V^{T}\left((\lambda-1) I_{n}+\frac{2 \tau \omega \lambda}{[(\alpha+\omega) \lambda-\alpha](2-\tau)} Q^{-1} B^{T} A^{-1} B\right) V\right|
$$$$
=\left|(\lambda-1) I_{n}+\frac{2 \tau \omega \lambda}{[(\alpha+\omega) \lambda-\alpha](2-\tau)} R\right|
$$$$
=\prod_{i=1}^{n}\left[(\lambda-1)+\frac{2 \tau \omega \lambda}{[(\alpha+\omega) \lambda-\alpha](2-\tau)} \mu_{i}\right]
$$$$
=(\lambda-1)^{k} \prod_{i=1}^{n-k}\left[(\lambda-1)+\frac{2 \tau \omega \lambda}{[(\alpha+\omega) \lambda-\alpha](2-\tau)} \mu_{i}\right] \text {; }
$$ 
that is,

$$
\begin{gathered}
\left|\lambda I-M_{\alpha, \omega, \tau}\right|=\left(\lambda-\frac{\alpha}{\alpha+\omega}\right)^{m}(\lambda-1)^{k} \\
\cdot \prod_{i=1}^{n-k}\left[\lambda-1+\frac{2 \tau \omega \lambda}{[(\alpha+\omega) \lambda-\alpha](2-\tau)} \mu_{i}\right] .
\end{gathered}
$$

Thus, $M_{\alpha, \omega, \tau}$ has at least $k$ eigenvalues 1 and $m$ eigenvalues $\alpha /(\alpha+\omega)$. After some calculations, we obtain (21) by $\lambda-$ $1+(2 \tau \omega \lambda /[(\alpha+\omega) \lambda-\alpha](2-\tau)) \mu_{i}=0$, which means that the remainder eigenvalues of $M_{\alpha, \omega, \tau}$ are the roots of (21). This proof is completed.

Lemma 9 (see [33]). index $\left(I-\Omega^{-1}(D-\Omega L)\right)=1$ holds if and only if, for any $0 \neq Y \in R(\mathscr{A}), Y \notin N\left(\mathscr{A}(D-\Omega L)^{-1} \Omega\right)$.

Lemma 10. Let $A$ and $Q$ be symmetric positive definite, let $B$ be rank deficient, and $\omega \neq 0$ and $\tau \neq 2$. Then, $\operatorname{index}\left(I-M_{\alpha, \omega, \tau}\right)=$ 1.

Proof. Let $X=\left(x^{T}, y^{T}\right)^{T}$, where $x \in \mathbb{R}^{m}$ and $y \in \mathbb{R}^{n}$ such that

$$
Y=\mathscr{A} X=\left(\begin{array}{cc}
A & B \\
-B^{T} & 0
\end{array}\right)\left(\begin{array}{l}
x \\
y
\end{array}\right)=\left(\begin{array}{c}
A x+B y \\
-B^{T} x
\end{array}\right) \neq 0 .
$$

To prove index $\left(I-M_{\alpha, \omega, \tau}\right)=1$, it is sufficient to prove $\mathscr{A} M_{1}^{-1} Y \neq 0$ by Lemma 9 , where $M_{1}^{-1}=(D-\Omega L)^{-1} \Omega$. Now we give the proof for $\mathscr{A} M_{1}^{-1} Y \neq 0$ by contradiction. Suppose $\mathscr{A} M_{1}^{-1} Y=0$. Let $U=\left(u^{T}, v^{T}\right)^{T}$ satisfy $\mathscr{A} U=0$, where $u \in \mathbb{R}^{m}$ and $v \in \mathbb{R}^{n}$. Then,

$$
\mathscr{A} U=\left(\begin{array}{cc}
A & B \\
-B^{T} & 0
\end{array}\right)\left(\begin{array}{l}
u \\
v
\end{array}\right)=\left(\begin{array}{l}
0 \\
0
\end{array}\right) \text {. }
$$

So we can get $A u+B v=0$ and $-B^{T} u=0$. From $A u+B v=0$, we obtain $u=-A^{-1} B v$, and $B^{T} A^{-1} B v=0$. Since $A$ is positive definite and so is $A^{-1}$, which results in $B v=0$, thus $u=0$. Hence,

$$
N(\mathscr{A})=\operatorname{span}\left\{\left(\begin{array}{l}
0 \\
\varphi
\end{array}\right)\right\}, \quad \text { where } B \varphi=0 .
$$

Then, there exists a vector $\varphi_{0}$ such that

$$
\begin{aligned}
M_{1}^{-1} Y & =\left(\begin{array}{l}
0 \\
\varphi_{0}
\end{array}\right), \\
B \varphi_{0} & =0,
\end{aligned}
$$

which yields that

$$
\begin{aligned}
Y & =M_{1}\left(\begin{array}{c}
0 \\
\varphi_{0}
\end{array}\right)=\left(\begin{array}{cc}
\frac{(\alpha+\omega)}{\omega} A & 0 \\
-B^{T} & \frac{2-\tau}{2 \tau} Q
\end{array}\right)\left(\begin{array}{l}
0 \\
\varphi_{0}
\end{array}\right) \\
& =\left(\begin{array}{c}
0 \\
\frac{2-\tau}{2 \tau} Q \varphi_{0}
\end{array}\right) .
\end{aligned}
$$

Making use of (38) and (42), we have $B \varphi_{0}=-(2 \tau /(2-$ $\tau)) B Q^{-1} B^{T} x=0$. Notice that $Q$ is positive definite, so is $Q^{-1}$; then $B^{T} x=0$, which implies that $\varphi_{0}=-(2 \tau /(2-\tau)) Q^{-1} B^{T} x=$ 0 . Hence,

$$
Y=M_{1}\left(\begin{array}{c}
0 \\
\varphi_{0}
\end{array}\right)=0
$$

which is a contradiction to $Y \neq 0$. Thus, $\mathscr{A} M_{1}^{-1} Y \neq 0$. This proof is completed.

By Lemma 10, we have proved that index $\left(I-M_{\alpha, \omega, \tau}\right)=1$, which satisfies the condition (1) in Lemma 7. Next, we need to prove the condition (2) in Lemma 7.

By Theorem 8 , we see that the eigenvalues of $M_{\alpha, \omega, \tau}$ are $1, \alpha /(\alpha+\omega)$ and the remainder eigenvalues are the roots of the quadratic equation (21). By Definition 6 and Lemma 7, we need to consider the eigenvalues of $M_{\alpha, \omega, \tau}$ except 1 .

Lemma 11. Let $A$ and $Q$ be symmetric positive definite, and let $B$ be rank deficient. Then, $\mathscr{V}\left(M_{\alpha, \omega, \tau}\right)<1$ for all $\alpha, \omega$, and $\tau$ such that

$$
\frac{\omega \tau \mu_{\max }}{2-\tau}<2 \alpha+\omega, \quad \omega>0, \alpha>0,0<\tau<2,
$$

where $\mu_{\text {max }}$ is the maximum eigenvalue of $Q^{-1} B^{T} A^{-1} B$.

Proof. Combining Lemma 2 and (21), we know that $\mathscr{V}\left(M_{\alpha, \omega, \tau}\right)<1$ if and only if

$$
\begin{aligned}
\left|\frac{\alpha}{\alpha+\omega}\right| & <1, \\
\left|\frac{2 \alpha+\omega}{\alpha+\omega}-\frac{2 \tau \omega \mu}{(\alpha+\omega)(2-\tau)}\right| & <\frac{\alpha}{\alpha+\omega}+1 .
\end{aligned}
$$

Using the same technique as the proof of Theorem 3, the conditions (44) are obtained. This completes our proof of Lemma 11.

Based on Definition 6 and Lemmas 10 and 11, we can establish the sufficient conditions of the semiconvergence for the GASOR method.

Theorem 12. Let $A$ and $Q$ be symmetric positive definite, and let $B$ be rank deficient. Then, the GASOR method is semiconvergent for all $\alpha, \omega$, and $\tau$ such that

$$
\frac{\omega \tau \mu_{\max }}{2-\tau}<2 \alpha+\omega, \quad \omega>0, \alpha>0,0<\tau<2,
$$

where $\mu_{\max }$ is the maximum nonzero eigenvalue of $Q^{-1} B^{T} A^{-1} B$.

\section{The Modified ASOR Method for Saddle Point Problems}

3.1. The Modified ASOR Method. To obtain the new iteration methods for saddle point problems, some authors added 
new parameters in the existing methods and got the better methods [11, 20, 53]. Based on the preconditioned HSS (PHSS) method derived by Bai et al. [2], Bai and Golub [20] and Li et al. [53] by using this method obtained the AHSS and parameterized preconditioned HSS (PPHSS) methods, respectively. This idea motivates us to propose the modified ASOR (MASOR) method for solving the saddle point problem (1) by making another splitting for the coefficient matrix of the saddle point problem (1) as follows:

$$
\mathscr{A}=D-L-U
$$

where

$$
D=\left(\begin{array}{cc}
\alpha A & 0 \\
0 & Q
\end{array}\right)
$$

$$
\begin{aligned}
L & =\left(\begin{array}{cc}
-A & 0 \\
B^{T} & \beta Q
\end{array}\right), \\
U & =\left(\begin{array}{cc}
\alpha A & -B \\
0 & (1-\beta) Q
\end{array}\right),
\end{aligned}
$$

with $\alpha>0$ and $Q \in \mathbb{R}^{n \times n}$ is a symmetric positive definite matrix. Let $\omega$ be nonzero real and let $\beta$ be real. Then, we consider the following modified ASOR iteration scheme for solving the saddle point problem (1):

$$
\left(\begin{array}{c}
x^{(k+1)} \\
y^{(k+1)}
\end{array}\right)=M_{\alpha, \beta, \omega}\left(\begin{array}{c}
x^{(k)} \\
y^{(k)}
\end{array}\right)+\omega(D-\omega L)^{-1}\left(\begin{array}{c}
p \\
-q
\end{array}\right),
$$

where $M_{\alpha, \beta, \omega}$ is the iteration matrix of the MASOR method and its form as follows:

$$
\begin{aligned}
& M_{\alpha, \beta, \omega}=(D-\omega L)^{-1}[(1-\omega) D+\omega U]=\left(\begin{array}{cc}
(\alpha+\omega) A & 0 \\
-\omega B^{T} & (1-\omega \beta) Q
\end{array}\right)^{-1}\left(\begin{array}{cc}
\alpha A & -\omega B \\
0 & (1-\omega \beta) Q
\end{array}\right) \\
& =\left(\begin{array}{cc}
\frac{1}{\alpha+\omega} A^{-1} & 0 \\
\frac{\omega}{(1-\omega \beta)(\alpha+\omega)} Q^{-1} B^{T} A^{-1} & \frac{1}{1-\omega \beta} Q^{-1}
\end{array}\right)\left(\begin{array}{cc}
\alpha A & -\omega B \\
0 & (1-\omega \beta) Q
\end{array}\right) \\
& =\left(\begin{array}{cc}
\frac{\alpha}{\alpha+\omega} I_{m} & -\frac{\omega}{\alpha+\omega} A^{-1} B \\
\frac{\alpha \omega}{(1-\omega \beta)(\alpha+\omega)} Q^{-1} B^{T} & I_{n}-\frac{\omega^{2}}{(1-\omega \beta)(\alpha+\omega)} Q^{-1} B^{T} A^{-1} B
\end{array}\right) .
\end{aligned}
$$

Then, the algorithmic description of the MASOR method is as follows.

The MASOR Method. Given initial vectors $x^{(0)} \in \mathbb{R}^{m}$ and $y^{(0)} \in \mathbb{R}^{n}$ and three real relaxation factors $\alpha>0, \omega \neq 0$, and $\beta$ is a real number, for $k=0,1,2, \ldots$, until the iteration sequence $\left\{\left(x^{(k)^{T}}\right),\left(y^{(k)^{T}}\right)\right\}$ converges to the exact solution of the saddle point problem (1), compute

$$
\begin{aligned}
& x^{(k+1)}=\frac{\alpha}{\alpha+\omega} x^{(k)}-\frac{\omega}{\alpha+\omega} A^{-1}\left(B y^{k}-p\right), \\
& y^{(k+1)}=y^{(k)}+\frac{\omega}{1-\omega \beta} Q^{-1}\left(B^{T} x^{(k+1)}-q\right) .
\end{aligned}
$$

Note that the MASOR method involves three parameters $\alpha, \omega$, and $\beta$. Since

$$
(D-\omega L)=\left(\begin{array}{cc}
(\alpha+\omega) A & 0 \\
-\omega B^{T} & (1-\omega \beta) Q
\end{array}\right)
$$

then the matrix $(D-\omega L)$ is invertible if and only if

$$
\begin{array}{r}
\alpha+\omega \neq 0, \\
\omega \beta \neq 1,
\end{array}
$$

by the definiteness of $A$ and $Q$.

Note that the MASOR method with $\beta=1 / 2$ reduces to the ASOR method proposed by Njeru and Guo [35].

\subsection{Convergence of the MASOR Method}

Lemma 13. Suppose that $\mu$ is an eigenvalue of matrix $Q^{-1} B^{T} A^{-1} B$, if $\lambda$ satisfies

$$
(\lambda-1)(1-\omega \beta)(\alpha-\alpha \lambda-\lambda \omega)=\lambda \omega^{2} \mu,
$$

and then $\lambda$ is an eigenvalue of iteration matrix $M_{\alpha, \beta, \omega}$. Conversely, if $\lambda$ is an eigenvalue of $M_{\alpha, \beta, \omega}$ such that $\lambda \neq 1, \omega \beta \neq 1$, $\lambda \neq \alpha /(\alpha+\omega)$, and $\mu$ satisfy (54), then $\mu$ is a nonzero eigenvalue of matrix $Q^{-1} B^{T} A^{-1} B$.

Proof. Since $Q^{-1} B^{T} A^{-1} B$ is nonsingular, $\mu \neq 0$. If $\lambda=0$ in (54), we obtain $0 \neq-\alpha(1-\omega \beta)=0=\lambda \omega^{2} \mu$, which is a 
contradiction. Thus, $\lambda \neq 0$. In a similar manner, we can prove that $\lambda \neq \alpha /(\alpha+\omega)$ and $\lambda \neq 1$.

We assume that $y$ is the eigenvector corresponding to $\mu$; thus, $y \neq 0$. Then by (54), the following holds:

$$
\frac{(\lambda-1)(1-\omega \beta)(\alpha-\alpha \lambda-\lambda \omega)}{\lambda \omega^{2}} y=\mu y,
$$

which yields that

$$
(\lambda-1)(1-\omega \beta) y=\frac{\lambda \omega^{2}}{\alpha-\alpha \lambda-\lambda \omega} Q^{-1} B^{T} A^{-1} B y .
$$

Let $x=(\omega /(\alpha-\alpha \lambda-\lambda \omega)) A^{-1} B y$; we can deduce that

$$
(\alpha-\alpha \lambda-\lambda \omega) A x=\omega B y
$$

and, by setting $x$ into (56), we have

$$
(\lambda-1)(1-\omega \beta) Q y=\lambda \omega B^{T} x .
$$

Combining (57) and (58) results in

$$
\frac{\omega}{(1-\omega \beta)(\alpha+\omega)} Q^{-1} B^{T}\left(\alpha x-\omega A^{-1} B y\right)=(\lambda-1) y .
$$

We rewrite (57) and (59) as follows:

$$
\begin{aligned}
& \frac{\alpha}{\alpha+\omega} x-\frac{\omega}{\alpha+\omega} A^{-1} B y=\lambda x, \\
& \frac{\alpha \omega}{(1-\omega \beta)(\alpha+\omega)} Q^{-1} B^{T} x+y \\
& -\frac{\omega^{2}}{(1-\omega \beta)(\alpha+\omega)} Q^{-1} B^{T} A^{-1} B y=\lambda y
\end{aligned}
$$

which is equivalent to

$$
\left(\begin{array}{cc}
\frac{\alpha}{\alpha+\omega} I_{m} & -\frac{\omega}{\alpha+\omega} A^{-1} B \\
\frac{\alpha \omega}{(1-\omega \beta)(\alpha+\omega)} Q^{-1} B^{T} & I_{n}-\frac{\omega^{2}}{(1-\omega \beta)(\alpha+\omega)} Q^{-1} B^{T} A^{-1} B
\end{array}\right)\left(\begin{array}{l}
x \\
y
\end{array}\right)=\lambda\left(\begin{array}{l}
x \\
y
\end{array}\right) .
$$

This implies that $M_{\alpha, \beta, \omega} \mathbf{x}=\lambda \mathbf{x}$, where $\mathbf{x}=\left(x^{T}, y^{T}\right)^{T} \neq 0$, which means that $\lambda$ is an eigenvalue of $M_{\alpha, \beta, \omega}$. We can prove the second assertion by revising the process.

Theorem 14. Let $A$ and $Q$ be symmetric positive definite, and let $B$ be full column rank. Assume that all eigenvalues $\mu$ of $Q^{-1} B^{T} A^{-1} B$ are real and positive. Then, the MASOR method is convergent for all $\alpha, \omega$, and $\tau$ such that

$$
\frac{\omega^{2} \mu_{\max }}{1-\omega \beta}<2(2 \alpha+\omega), \quad \omega>0, \alpha>0,1-\omega \beta>0,
$$

where $\mu_{\max }$ is the maximum eigenvalue of $Q^{-1} B^{T} A^{-1} B$.

Proof. Equation (54) can be equivalently written as

$$
\lambda^{2}+\left(\frac{\omega^{2} \mu}{(\alpha+\omega)(1-\omega \beta)}-\frac{2 \alpha+\omega}{\alpha+\omega}\right) \lambda+\frac{\alpha}{\alpha+\omega}=0 .
$$

Equation (63) can be expressed as $x^{2}-b x+c=0$, where

$$
\begin{aligned}
& b=\frac{2 \alpha+\omega}{\alpha+\omega}-\frac{\omega^{2} \mu}{(\alpha+\omega)(1-\omega \beta)}, \\
& c=\frac{\alpha}{\alpha+\omega} .
\end{aligned}
$$

Applying Lemma 2 to the quadratic equation (63), we know that $|\lambda|<1$ if and only if

$$
\begin{aligned}
\left|\frac{\alpha}{\alpha+\omega}\right| & <1, \\
\left|\frac{2 \alpha+\omega}{\alpha+\omega}-\frac{\omega^{2} \mu}{(\alpha+\omega)(1-\omega \beta)}\right| & <\frac{\alpha}{\alpha+\omega}+1 .
\end{aligned}
$$

In terms of (65), we derive

$$
\begin{aligned}
\omega & >0, \\
-2 \alpha & <\omega, \\
0 & <\frac{\omega^{2} \mu}{1-\omega \beta}<2(2 \alpha+\omega) .
\end{aligned}
$$

Evidently, the first and the second inequalities in (66) hold true for all $\alpha>0$ and $\omega>0$. It follows from the third inequality in (66), $\omega^{2}>0$, and $\mu$ which is a real and positive eigenvalue of the matrix $Q^{-1} B^{T} A^{-1} B$ that

$$
\begin{aligned}
& 1-\omega \beta>0, \\
& \frac{\omega^{2} \mu}{1-\omega \beta}<2(2 \alpha+\omega) .
\end{aligned}
$$

It is not difficult to verify that

$$
\frac{\omega^{2} \mu}{1-\omega \beta} \leq \frac{\omega^{2} \mu_{\max }}{1-\omega \beta}
$$

Therefore, the proof of this theorem is completed. 
From (63), we get the following corollary.

Corollary 15. Let $A$ and $Q$ be symmetric positive definite, and let $B$ be be full rank. If $\mu$ is an eigenvalue of the matrix $Q^{-1} B^{T} A^{-1} B$, then eigenvalues of the matrix $M_{\alpha, \beta, \omega}$ are given by $\lambda=\alpha /(\alpha+\omega)$ or

$$
\begin{aligned}
\lambda= & \frac{1}{2}\left[\left(\frac{2 \alpha+\omega}{\alpha+\omega}-\frac{\omega^{2} \mu}{(\alpha+\omega)(1-\omega \beta)}\right)\right. \\
& \left. \pm \sqrt{\left(\frac{\omega^{2} \mu}{(\alpha+\omega)(1-\omega \beta)}-\frac{2 \alpha+\omega}{\alpha+\omega}\right)^{2}-\frac{4 \alpha}{\alpha+\omega}}\right] .
\end{aligned}
$$

$$
\left|\lambda I-M_{\alpha, \beta, \omega}\right|=\left|\begin{array}{cc}
\left(\lambda-\frac{\alpha}{\alpha+\omega}\right) I_{m} & \frac{\omega}{\alpha+\omega} A^{-1} B \\
-\frac{\alpha \omega}{(1-\omega \beta)(\alpha+\omega)} Q^{-1} B^{T} & (\lambda-1) I_{n}+\frac{\omega^{2}}{(1-\omega \beta)(\alpha+\omega)} Q^{-1} B^{T} A^{-1} B
\end{array}\right| .
$$

If $\lambda=\alpha /(\alpha+\omega)$, with the similar manner applied in the proof of Theorem 8, we can prove that $\left|\lambda I-M_{\alpha, \beta, \omega}\right|=0$, which
3.3. Semiconvergence of the MASOR Method for Singular Saddle Point Problem. In this section, we assume that $B$ in (1) is rank deficient and we will discuss the semiconvergence region for parameters $\omega, \alpha$, and $\beta$ in the MASOR method for solving the singular saddle point problem (1).

Theorem 16. Let $A$ and $Q$ be symmetric positive definite, and let $B$ be rank deficient. If $\mu$ is an eigenvalue of the matrix $Q^{-1} B^{T} A^{-1} B$, then eigenvalues of the matrix $M_{\alpha, \beta, \omega}$ are $1, \lambda=$ $\alpha /(\alpha+\omega)$, and the remainder eigenvalues are the roots of the quadratic equation (63).

Proof. By simple calculations, we have means that $\alpha /(\alpha+\omega)$ is an eigenvalue of $M_{\alpha, \beta, \omega}$. Otherwise, $\lambda \neq \alpha /(\alpha+\omega)$, after suitable manipulations, gives

$$
\begin{aligned}
\left|\lambda I-M_{\alpha, \beta, \omega}\right| & =\left|\begin{array}{cc}
\left(\lambda-\frac{\alpha}{\alpha+\omega}\right) I_{m} & \frac{\omega}{\alpha+\omega} A^{-1} B \\
0 & (\lambda-1) I_{n}+\frac{\omega^{2} \lambda}{[(\alpha+\omega) \lambda-\alpha](1-\omega \beta)} Q^{-1} B^{T} A^{-1} B
\end{array}\right| \\
& =\left(\lambda-\frac{\alpha}{\alpha+\omega}\right)^{m}\left|\begin{array}{cc}
I_{m} & \frac{\omega}{\alpha+\omega} A^{-1} B \\
0 & (\lambda-1) I_{n}+\frac{\omega^{2} \lambda}{[(\alpha+\omega) \lambda-\alpha](1-\omega \beta)} Q^{-1} B^{T} A^{-1} B
\end{array}\right| \\
& =\left(\lambda-\frac{\alpha}{\alpha+\omega}\right)^{m}\left|(\lambda-1) I_{n}+\frac{\omega}{[(\alpha+\omega) \lambda-\alpha](1-\omega \beta)} Q^{-1} B^{T} A^{-1} B\right| .
\end{aligned}
$$

We assume that $\operatorname{rank}(B)=n-k(k>0)$ and, with a quite similar strategy utilized in Theorem 8 , we deduce that 
Lemma 17. Let $A$ and $Q$ be symmetric positive definite, let $B$ be rank deficient, and $\omega \neq 0$ and $\omega \neq 1 / \beta$. Then, index $(I-$ $\left.M_{\alpha, \beta, \omega}\right)=1$.

Proof. We prove this theorem by Lemma 9. Let $X=\left(x^{T}\right.$, $\left.y^{T}\right)^{T}$, with $x \in \mathbb{R}^{m}$ and $y \in \mathbb{R}^{n}$ such that

$$
Y=\mathscr{A} X=\left(\begin{array}{cc}
A & B \\
-B^{T} & 0
\end{array}\right)\left(\begin{array}{l}
x \\
y
\end{array}\right)=\left(\begin{array}{c}
A x+B y \\
-B^{T} x
\end{array}\right) \neq 0 .
$$

In order to prove index $\left(I-M_{\alpha, \beta, \omega}\right)=1$, it remains only to prove $\mathscr{A} M_{2}^{-1} Y \neq 0$, where $M_{2}^{-1}=\omega(D-\omega L)^{-1}$. Here and in the sequel, we prove $\mathscr{A} M_{2}^{-1} Y \neq 0$ by contradiction. We assume that $\mathscr{A} M_{2}^{-1} Y=0$. Let $F=\left(f^{T}, g^{T}\right)^{T}$, where $f \in \mathbb{R}^{m}$, $g \in \mathbb{R}^{n}$, and $\mathscr{A F}=0$. That is

$$
\mathscr{A} U=\left(\begin{array}{cc}
A & B \\
-B^{T} & 0
\end{array}\right)\left(\begin{array}{l}
f \\
g
\end{array}\right)=\left(\begin{array}{l}
0 \\
0
\end{array}\right)
$$

which results in $A f+B g=0$ and $-B^{T} f=0$. From $A f+B g=$ 0 , we deduce that $f=-A^{-1} B g$ and $B^{T} A^{-1} B g=0$. From the fact that $A$ is positive definite and so is $A^{-1}$, which leads to $B g=0$, thus $f=0$. Then, we infer that

$$
N(\mathscr{A})=\operatorname{span}\left\{\left(\begin{array}{l}
0 \\
\varphi
\end{array}\right)\right\}, \quad \text { where } B \varphi=0 .
$$

Therefore, there is a vector $\varphi_{0}$ such that

$$
\begin{aligned}
M_{2}^{-1} Y & =\left(\begin{array}{c}
0 \\
\varphi_{0}
\end{array}\right), \\
B \varphi_{0} & =0 .
\end{aligned}
$$

It follows from (76) that

$$
\begin{aligned}
Y & =M_{2}\left(\begin{array}{c}
0 \\
\varphi_{0}
\end{array}\right)=\left(\begin{array}{cc}
\frac{\alpha+\omega}{\omega} A & 0 \\
-B^{T} & \frac{1-\omega \beta}{\omega} Q
\end{array}\right)\left(\begin{array}{l}
0 \\
\varphi_{0}
\end{array}\right) \\
& =\left(\begin{array}{c}
0 \\
\frac{1-\omega \beta}{\omega} Q \varphi_{0}
\end{array}\right) .
\end{aligned}
$$

Combining (73) with (77) gives $B \varphi_{0}=-(\omega /(1-$ $\omega \beta)) B Q^{-1} B^{T} x=0 . Q^{-1}$ is positive definite as $Q$ is positive definite, and thus $B^{T} x=0$, which results in $\varphi_{0}=-(\omega /(1-$ $\omega \beta)) Q^{-1} B^{T} x=0$. Then, $Y=0$ holds, which is in contradiction with (73). So $\mathscr{A} M_{2}^{-1} Y \neq 0$. From the above analysis and Lemma 9, it has index $\left(I-M_{\alpha, \beta, \omega}\right)=1$. This proves the theorem.

By Lemma 17, we have proved that index $\left(I-M_{\alpha, \beta, \omega}\right)=1$, which satisfies the condition (1) in Lemma 7. In the sequel, we need to prove condition (2) in Lemma 7.

By Theorem 16, we see that the eigenvalues of $M_{\alpha, \beta, \omega}$ are $1, \alpha /(\alpha+\omega)$ and the remainder eigenvalues are the roots of the quadratic equation (63). By Definition 6 and Lemma 7, we only consider the eigenvalues of $M_{\alpha, \beta, \omega}$ except 1 .
Lemma 18. Let $A$ and $Q$ be symmetric positive definite, and let $B$ be rank deficient. Then, $\mathscr{V}\left(M_{\alpha, \beta, \omega}\right)<1$ for all $\alpha, \beta$, and $\omega$ such that

$$
\frac{\omega^{2} \mu_{\max }}{1-\omega \beta}<2(2 \alpha+\omega), \quad \omega>0, \alpha>0,1-\omega \beta>0,
$$

where $\mu_{\max }$ is the maximum eigenvalue of $Q^{-1} B^{T} A^{-1} B$.

Together with Definition 6 and Lemmas 17 and 18, we get the following sufficient conditions for the semiconvergence of the MASOR method.

Theorem 19. Let $A$ and $Q$ be symmetric positive definite, and let $B$ be rank deficient. Then, the MASOR method is semiconvergent for all $\alpha, \beta$, and $\omega$ such that

$$
\frac{\omega^{2} \mu_{\max }}{1-\omega \beta}<2(2 \alpha+\omega), \quad \omega>0, \alpha>0,1-\omega \beta>0,
$$

where $\mu_{\max }$ is the maximum nonzero eigenvalue of $Q^{-1} B^{T} A^{-1} B$.

\section{The Optimal Convergence Factors of the GASOR and MASOR Methods}

According to the theory of iterative methods, the optimal parameters of the GASOR method and MASOR method are as follows:

$$
\begin{aligned}
& \left(\alpha^{*}, \omega^{*}, \tau^{*}\right)=\arg \min _{(\alpha, \omega, \tau)} \rho\left(M_{\alpha, \omega, \tau}\right), \\
& \left(\alpha^{*}, \beta^{*}, \omega^{*}\right)=\arg \min _{(\alpha, \beta, \omega)} \rho\left(M_{\alpha, \beta, \omega}\right),
\end{aligned}
$$

respectively, where the $\arg \min _{(\alpha, \beta, \omega)} \rho\left(L_{\alpha, \beta, \omega}\right)$ denotes such $\alpha$, $\beta$, and $\omega$ that the spectral radius of the matrix $L_{\alpha, \beta, \omega}$ reaches the minimum.

This problem has been discussed in some articles $[2,5$, $8,10,12,19]$. Golub et al. [8] obtained the optimal parameter of the SOR-like method; Bai et al. and Li and Kong studied the optimal parameters of the GSOR-like and GSOR methods in [5, 10], respectively. Bai [19] discussed the optimal parameters of the HSS-like method and Bai et al. [2] studied the optimal parameters of the PHSS method and so forth. To get the optimal values of $\alpha, \omega$, and $\tau$ for the GASOR method and $\alpha, \beta$, and $\omega$ for the MASOR method, we need to analyze the modulus of eigenvalues of $M_{\alpha, \omega, \tau}$ and $M_{\alpha, \beta, \omega}$, respectively. Based on the proof of Theorems 8 and 16, the matrices $M_{\alpha, \omega, \tau}$ and $M_{\alpha, \beta, \omega}$ have $m$ repeated eigenvalues $\alpha /(\alpha+\omega)$ and the remainder eigenvalues satisfy (21) and (63), respectively. Note that all the eigenvalues $\lambda$ of $M_{\alpha, \omega, \tau}$ and $M_{\alpha, \beta, \omega}$ are available, which depend on $\alpha, \beta, \omega$, and $\tau$. The optimal parameters could be obtained by getting the minimum of these eigenvalue functions. However, for most iterative methods, especially with multiple parameters, this work is very complicated. Hence it is very difficult to get the optimal parameters. The parameters in this paper are chosen based on prior experience and trial and error. 


\section{Numerical Experiments}

In this section, numerical examples illustrate the superiority of the GASOR and MASOR methods to the ASOR, SORlike, GSOR, and MSOR-like methods when they are used for solving the nonsingular saddle point problem (1) and show the advantages of the GASOR and MASOR methods over the GSOR, GSSOR, MSSOR, and GMSSOR methods for solving the singular saddle point problem (1). All numerical procedures are carried out using Matlab 6.5 on a personal computer with Intel(R) Pentium(R) CPU G3240T 2.70 GHz, 2.0 G memory and Windows 7 operating system.

Example 1. Consider the Stokes flow problem [2]: find $\mathbf{u}$ and w such that

$$
\begin{array}{rlrl}
-\mu \Delta \mathbf{u}+\nabla \mathbf{w}=\tilde{f}, & & \text { in } \Omega, \\
\nabla \cdot \mathbf{u}=\tilde{g}, & & \text { in } \Omega, \\
\mathbf{u}=0, & \text { on } \partial \Omega, \\
\int_{\Omega} \mathbf{w}(x) d x=0, &
\end{array}
$$

where $\Omega=(0,1) \times(0,1) \subset \mathbb{R}^{2}, \partial \Omega$ is the boundary of $\Omega, \mu$ stands for the viscosity scalar, $\mathbf{u}=\left(u_{1}^{T}, u_{2}^{T}\right)^{T}$ is a vector-valued function representing the velocity, $\Delta$ is the componentwise Laplace operator, and $\mathbf{w}$ is a scalar function representing the pressure. By discretizing (82) with the upwind scheme as follows [54, 55]:

$$
\begin{aligned}
& \frac{\mu}{h^{2}}\left(4 u_{1}^{i, j}-u_{1}^{i, j+1}-u_{1}^{i, j-1}-u_{1}^{i+1, j}-u_{1}^{i-1, j}\right) \\
& \quad+\frac{1}{h}\left(\mathbf{w}^{i, j}-\mathbf{w}^{i-1, j}\right)=f_{1}, \\
& \frac{\mu}{h^{2}}\left(4 u_{2}^{i, j}-u_{2}^{i, j+1}-u_{2}^{i, j-1}-u_{2}^{i+1, j}-u_{2}^{i-1, j}\right) \\
& \quad+\frac{1}{h}\left(\mathbf{w}^{i, j}-\mathbf{w}^{i, j-1}\right)=f_{2}, \\
& -\frac{1}{h}\left(u_{1}^{i, j}-u_{1}^{i-1, j}\right)-\frac{1}{h}\left(u_{2}^{i, j}-u_{2}^{i, j-1}\right)=-g,
\end{aligned}
$$

we obtain the system

$$
\left(\begin{array}{cc}
A & B \\
-B^{T} & 0
\end{array}\right)\left(\begin{array}{c}
\mathbf{u} \\
\mathbf{w}
\end{array}\right)=\left(\begin{array}{c}
f \\
-g
\end{array}\right)
$$

where

$$
\begin{aligned}
A & =\left(\begin{array}{cc}
I \otimes T+T \otimes I & 0 \\
0 & I \otimes T+T \otimes I
\end{array}\right) \in \mathbb{R}^{2 p^{2} \times 2 p^{2}}, \\
B & =\left(\begin{array}{l}
I \otimes F \\
F \otimes I
\end{array}\right) \in \mathbb{R}^{2 p^{2} \times p^{2}},
\end{aligned}
$$

with

$$
\begin{aligned}
& T=\frac{\mu}{h^{2}} \cdot \operatorname{tridiag}(-1,2,-1) \in \mathbb{R}^{p \times p}, \\
& F=\frac{1}{h} \cdot \operatorname{tridiag}(-1,1,0) \in \mathbb{R}^{p \times p} .
\end{aligned}
$$

TABLE 1: Choice of the matrix $Q$.

\begin{tabular}{lcc}
\hline Case number & Matrix $Q$ & Description \\
\hline 1 & $B^{T} \widehat{A}^{-1} B$ & $\widehat{A}=\operatorname{tridiag}(A)$ \\
2 & $B^{T} \widehat{A}^{-1} B$ & $\widehat{A}=\operatorname{diag}(A)$ \\
\hline
\end{tabular}

Here $\otimes$ denotes the Kronecker product, $h=1 /(p+1)$ is the discretization mesh size, and $J=\operatorname{tridiag}(a, b, c)$ denotes a tridiagonal matrix with $J_{i+1, i}=a, J_{i, i}=b$, and $J_{i, i+1}=$ $c$. In addition, we choose the right-hand side vector $b$ so that the exact solution of the saddle point problem (1) is $(1,1, \ldots, 1)^{T} \in \mathbb{R}^{m+n}$, where $m=2 p^{2}$ and $n=p^{2}$. The preconditioning matrix $Q$ is an approximation of matrix $Q=$ $B^{T} A^{-1} B$. Moreover, we choose $\mu=1$ in this example. We consider two cases as listed in Table 1.

All computations for the SOR-like, GSOR, MSOR-like, ASOR, GASOR, and MASOR methods are started from initial vector $\mathbf{x}^{(0)}=\left(x^{(0) T}, y^{(0) T}\right)^{T}=(0,0, \ldots, 0)^{T}$ and the iteration is terminated once the current iterate $\mathbf{x}^{(k)}$ satisfies

$$
\begin{aligned}
\mathrm{RES} & =\frac{\sqrt{\left\|f-A x^{(k)}-B y^{(k)}\right\|_{2}^{2}+\left\|g-B^{*} x^{(k)}\right\|_{2}^{2}}}{\sqrt{\|f\|_{2}^{2}+\|g\|_{2}^{2}}} \\
& <10^{-9},
\end{aligned}
$$

or the maximum prescribed number of iterations $k_{\max }=15 p$ is exceeded.

In Table 2, for various problem sizes $p$, we list the theoretical optimal iteration parameters of the SOR-like, GSOR, MSOR-like, ASOR, GASOR, and MASOR methods used in our implementations. For the GSOR, SOR-like, and MSORlike methods, we adopt their optimal parameters as in $[5,8$, 9]. In addition, the parameters taken by the ASOR method are the same as in [35], and we choose the parameters of the GASOR and MASOR methods which result in the least numbers of iterations for this numerical example. However, note that the explicit expressions of parameters cannot be obtained, and we only choose them by trial and error. The corresponding convergence factors $\rho^{*}, \rho_{\exp }$, and $\rho$ with various problems size $p$ are also reported in Table 2 .

In Table 3, we present the iteration numbers (IT), CPU times (CPU), and relative residual (RES) of the testing iteration methods with different problem sizes $p$. In this table, we use the bold numbers to indicate the smallest and the second smallest CPU times and iteration numbers in each column.

As observed in Table 2, the convergence factors of the GASOR and MASOR methods are smaller than that of the SOR-like method. We can also see that the spectral radii of the GASOR and MASOR methods in Cases 1 and 2 for this example are almost the same as the ones of the GSOR, MSORlike and ASOR methods, while the GASOR and MASOR methods require much less time and iteration steps to achieve the stopping criterion than other four methods, and the relaxation parameters of the GASOR and MASOR methods are not optimal and only lie in the convergence region of these methods. From the results in Table 3, we see that the SOR-like, GSOR, MSOR-like, ASOR, GASOR, and MASOR methods 
TABLE 2: Parameters for the six methods and the corresponding spectral radii.

\begin{tabular}{|c|c|c|c|c|}
\hline Method & $p$ & 16 & 24 & 32 \\
\hline \multirow{4}{*}{ SOR-like } & & Case 1 & & \\
\hline & $\omega^{*}$ & 0.3657 & 0.2620 & 0.2037 \\
\hline & $\rho^{*}$ & 0.7964 & 0.8591 & 0.8923 \\
\hline & $\omega^{*}$ & 0.4429 & 0.3307 & 0.2635 \\
\hline \multirow[t]{3}{*}{ GSOR } & $\tau^{*}$ & 0.2854 & 0.1985 & 0.1519 \\
\hline & $\rho^{*}$ & 0.7464 & 0.8181 & 0.8582 \\
\hline & $\omega^{*}$ & 0.2498 & 0.1806 & 0.1412 \\
\hline \multirow[t]{3}{*}{ MSOR-like } & $\alpha^{*}$ & 1.7732 & 1.8315 & 1.8662 \\
\hline & $\rho^{*}$ & 0.7464 & 0.8181 & 0.8582 \\
\hline & $\omega_{\exp }$ & 0.2531 & 0.1817 & 0.1417 \\
\hline \multirow[t]{3}{*}{ ASOR } & $\alpha_{\exp }$ & 0.3300 & 0.3944 & 0.4401 \\
\hline & $\rho_{\exp }$ & 0.7523 & 0.8274 & 0.8697 \\
\hline & $\alpha_{\exp }$ & 0.4117 & 0.3697 & 0.2799 \\
\hline \multirow{4}{*}{ GASOR } & $\omega_{\exp }$ & 0.3200 & 0.1820 & 0.1000 \\
\hline & $\tau_{\exp }$ & 0.2450 & 0.1780 & 0.1400 \\
\hline & $\rho$ & 0.7657 & 0.8373 & 0.8701 \\
\hline & $\omega_{\exp }$ & 0.2531 & 0.1820 & 0.1002 \\
\hline \multirow{3}{*}{ MASOR } & $\alpha_{\exp }$ & 0.319 & 0.3698 & 0.2800 \\
\hline & $\beta_{\exp }$ & 0.3900 & 0.3769 & 3.3550 \\
\hline & $\rho$ & 0.7737 & 0.8371 & 0.8690 \\
\hline \multirow{4}{*}{ SOR-like } & & Case 2 & & \\
\hline & $\omega^{*}$ & 0.2720 & 0.1915 & 0.1476 \\
\hline & $\rho^{*}$ & 0.8533 & 0.8992 & 0.9232 \\
\hline & $\omega^{*}$ & 0.3419 & 0.2489 & 0.1956 \\
\hline \multirow[t]{3}{*}{ GSOR } & $\tau^{*}$ & 0.2066 & 0.1423 & 0.1084 \\
\hline & $\rho^{*}$ & 0.8112 & 0.8667 & 0.8969 \\
\hline & $\omega^{*}$ & 0.1873 & 0.1328 & 0.1029 \\
\hline \multirow[t]{3}{*}{ MSOR-like } & $\alpha^{*}$ & 1.8256 & 1.8737 & 1.9009 \\
\hline & $\rho^{*}$ & 0.8112 & 0.8667 & 0.8969 \\
\hline & $\omega_{\exp }$ & 0.1886 & 0.1333 & 0.1031 \\
\hline \multirow[t]{3}{*}{ ASOR } & $\alpha_{\exp }$ & 0.3873 & 0.4509 & 0.4934 \\
\hline & $\rho_{\exp }$ & 0.8201 & 0.8785 & 0.9095 \\
\hline & $\alpha_{\exp }$ & 0.4400 & 0.4000 & 0.4150 \\
\hline \multirow{4}{*}{ GASOR } & $\omega_{\exp }$ & 0.2400 & 0.1348 & 0.1040 \\
\hline & $\tau_{\exp }$ & 0.1780 & 0.1280 & 0.1000 \\
\hline & $\rho$ & 0.8590 & 0.8942 & 0.9181 \\
\hline & $\omega_{\exp }$ & 0.1886 & 0.1333 & 0.1031 \\
\hline \multirow{3}{*}{ MASOR } & $\alpha_{\exp }$ & 0.3450 & 0.3900 & 0.414 \\
\hline & $\beta_{\exp }$ & 0.2090 & 0.2481 & 0.2100 \\
\hline & $\rho$ & 0.8579 & 0.8941 & 0.9171 \\
\hline
\end{tabular}

succeed in achieving the stopping criterion within the largest admissible number of iteration steps in all cases. Moreover, the GASOR method uses the least iteration numbers and CPU times compared with the SOR-like, GSOR, MSOR-like, ASOR, and MASOR methods, and the MASOR method is also superior to the SOR-like, GSOR, MSOR-like, and ASOR methods both in iteration numbers and CPU times especially for large problem size $m$. Furthermore, we can find that the
TABLE 3: Numerical results for the six methods for Case 1 and Case 2 , respectively.

\begin{tabular}{|c|c|c|c|c|}
\hline Method & $p$ & 16 & 24 & 32 \\
\hline \multicolumn{5}{|c|}{ Case 1} \\
\hline \multirow{3}{*}{ SOR-like } & IT & 131 & 201 & 273 \\
\hline & CPU & 0.3793 & 2.8314 & 10.8478 \\
\hline & RES & $8.76 e-010$ & $8.78 e-010$ & $9.14 e-010$ \\
\hline \multirow{3}{*}{ GSOR } & IT & 100 & 149 & 200 \\
\hline & CPU & 0.2947 & 2.0845 & 7.7318 \\
\hline & RES & $8.87 e-010$ & $9.86 e-010$ & $9.41 e-010$ \\
\hline \multirow{3}{*}{ MSOR-like } & IT & 99 & 149 & 201 \\
\hline & $\mathrm{CPU}$ & 0.2813 & 2.0790 & 7.6604 \\
\hline & RES & $7.90 e-010$ & $9.49 e-010$ & $8.82 e-010$ \\
\hline \multirow{3}{*}{ ASOR } & IT & 87 & 127 & 172 \\
\hline & $\mathrm{CPU}$ & 0.2559 & 1.7806 & 6.5416 \\
\hline & RES & $1.25 e-010$ & $7.52 e-010$ & $5.29 e-010$ \\
\hline \multirow{3}{*}{ GASOR } & IT & 80 & 116 & 154 \\
\hline & $\mathrm{CPU}$ & 0.2279 & 1.6303 & 5.8605 \\
\hline & RES & $6.26 e-010$ & $5.14 e-010$ & $9.07 e-010$ \\
\hline \multirow{3}{*}{ MASOR } & IT & 83 & 116 & 157 \\
\hline & $\mathrm{CPU}$ & 0.2396 & 1.6388 & 6.0598 \\
\hline & RES & $5.69 e-010$ & $6.85 e-010$ & $7.75 e-010$ \\
\hline \multicolumn{5}{|c|}{ Case 2} \\
\hline \multirow{3}{*}{ SOR-like } & IT & 192 & 292 & 389 \\
\hline & $\mathrm{CPU}$ & 0.5437 & 4.2177 & 15.4625 \\
\hline & RES & $9.81 e-010$ & $9.27 e-010$ & $9.91 e-010$ \\
\hline \multirow{3}{*}{ GSOR } & IT & 143 & 215 & 287 \\
\hline & $\mathrm{CPU}$ & 0.4612 & 3.0769 & 11.1054 \\
\hline & RES & $8.75 e-010$ & $9.87 e-010$ & $9.15 e-010$ \\
\hline \multirow{3}{*}{ MSOR-like } & IT & 143 & 213 & 286 \\
\hline & CPU & 0.4110 & 2.9643 & 11.0999 \\
\hline & RES & $9.36 e-010$ & $9.83 e-010$ & $9.17 e-010$ \\
\hline \multirow{3}{*}{ ASOR } & IT & 125 & 185 & 261 \\
\hline & CPU & 0.3571 & 2.5767 & 10.1672 \\
\hline & RES & $9.80 e-010$ & $9.56 e-010$ & $5.48 e-010$ \\
\hline \multirow{3}{*}{ GASOR } & IT & 108 & 160 & 219 \\
\hline & $\mathrm{CPU}$ & 0.3067 & 2.2436 & 8.4094 \\
\hline & RES & $3.83 e-010$ & $8.29 e-010$ & $8.85 e-010$ \\
\hline \multirow{3}{*}{ MASOR } & IT & 110 & 161 & 226 \\
\hline & $\mathrm{CPU}$ & 0.3123 & 2.3058 & 8.7687 \\
\hline & RES & $9.62 e-010$ & $9.97 e-010$ & $4.51 e-010$ \\
\hline
\end{tabular}

performance of the GASOR and MASOR methods is almost the same.

In order to better understand the numerical results, we have presented graphs of RES $(\log 10)$ against number of iterations in Table 3 for $p=16, p=24$, and $p=32$, respectively, in Figures 1-3. From the three figures, we note that the six methods are convergent while the GASOR and MASOR methods converge faster than other methods. Moreover, it can be seen that the GASOR and MASOR methods at different iteration points have "semilocal convergence" behavior, and thus we can deduce that the parameters chosen by the 

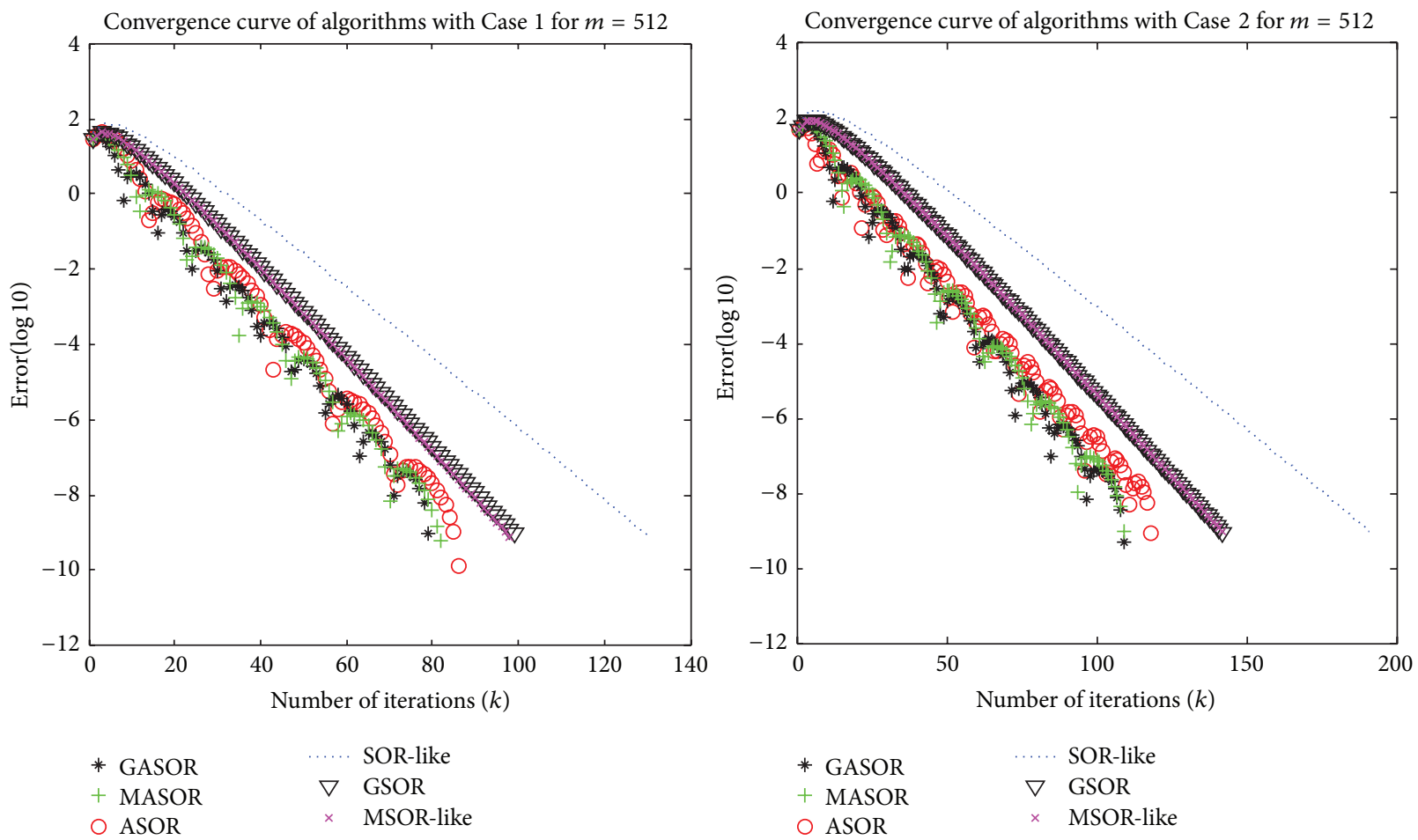

FIGURE 1: The iteration curves of algorithms when $m=512$ for Case 1 and Case 2, respectively.
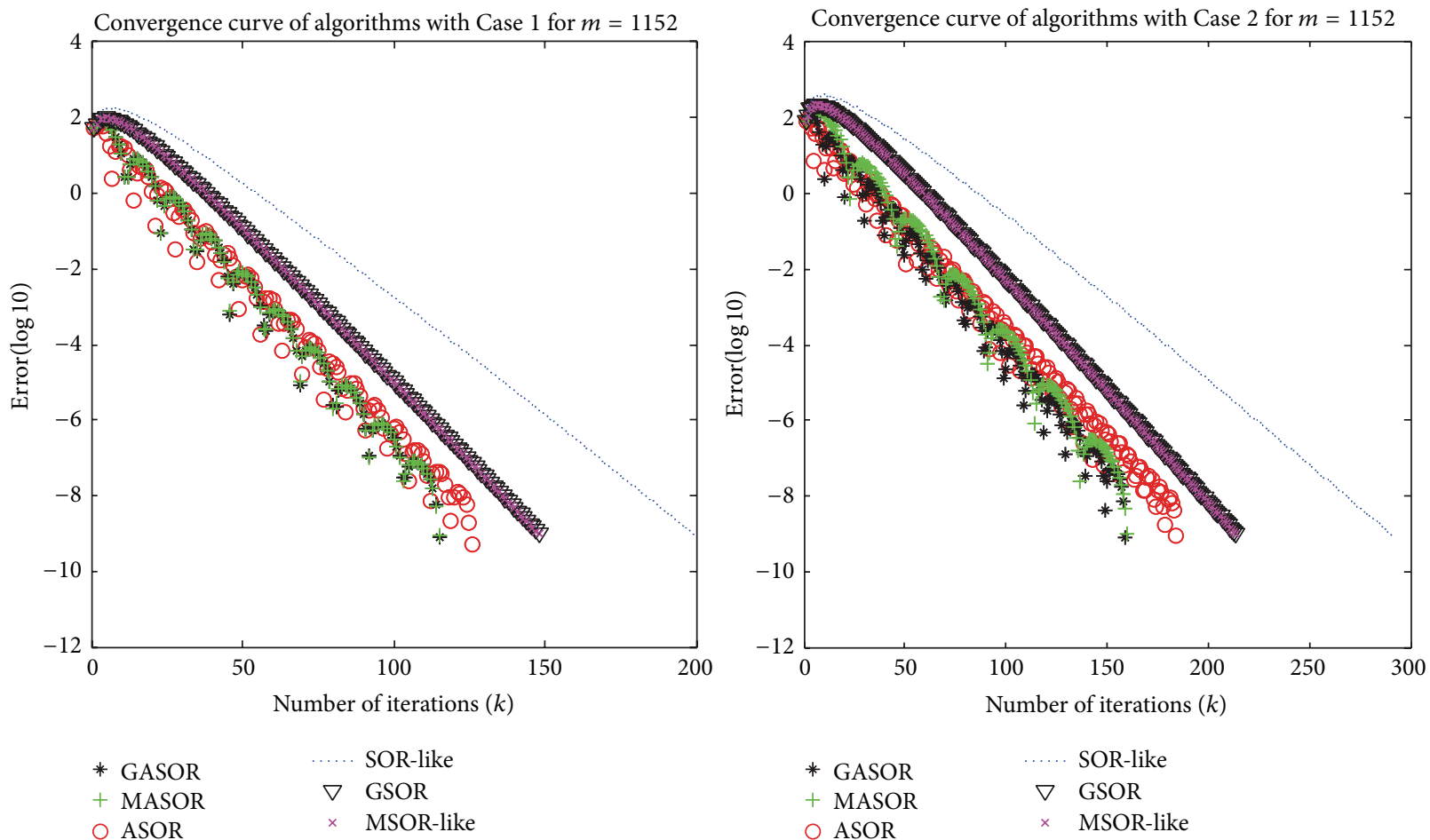

FIGURE 2: The iteration curves of algorithms when $m=1152$ for Case 1 and Case 2, respectively. 

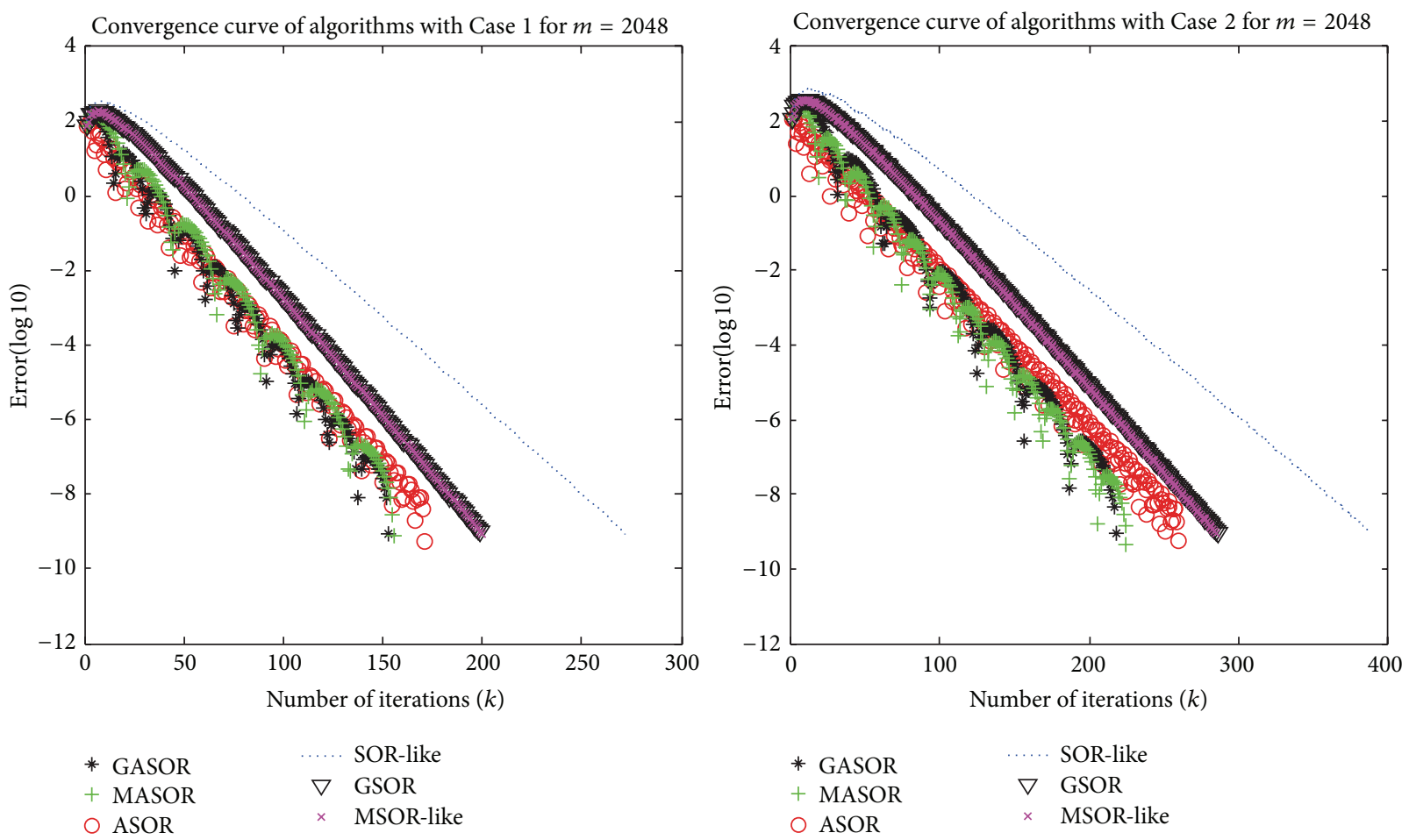

FIGURE 3: The iteration curves of algorithms when $m=2048$ for Case 1 and Case 2, respectively.

GASOR and MASOR methods are not optimal and only lie in the convergence regions of these two new methods, which means that the GASOR and MASOR methods may perform better with better choice of optimal parameters. The numerical results in this example show feasibility and effectiveness of the GASOR and MASOR methods for solving nonsingular saddle point problems.

Example 2. Consider the singular saddle point problem (1) with coefficient matrix of the matrix blocks [34]

$$
\begin{aligned}
& A=\left(\begin{array}{cc}
I \otimes T+T \otimes I & 0 \\
0 & I \otimes T+T \otimes I
\end{array}\right) \in \mathbb{R}^{2 p^{2} \times 2 p^{2}}, \\
& B=\left(\begin{array}{lll}
\widehat{B} & b_{1} & b_{2}
\end{array}\right) \in \mathbb{R}^{2 p^{2} \times\left(p^{2}+2\right)},
\end{aligned}
$$

where

$$
\begin{aligned}
& T=\frac{1}{h^{2}} \cdot \operatorname{tridiag}(-1,2,-1) \in \mathbb{R}^{p \times p}, \\
& \widehat{B}=\left(\begin{array}{l}
I \otimes F \\
F \otimes I
\end{array}\right) \in \mathbb{R}^{2 p^{2} \times p^{2}}, \\
& b_{1}=\widehat{B}\left(\begin{array}{l}
e \\
0
\end{array}\right),
\end{aligned}
$$

$$
\begin{aligned}
& b_{2}=\widehat{B}\left(\begin{array}{l}
0 \\
e
\end{array}\right), \quad e=(1,1, \ldots, 1) \in \mathbb{R}^{p^{2} / 2}, \\
& F=\frac{1}{h} \cdot \operatorname{tridiag}(-1,1,0) \in \mathbb{R}^{p \times p}, \quad h=\frac{1}{p+1},
\end{aligned}
$$

and the right-hand side vector $b$ is chosen by $b=\mathscr{A} e_{m+n}$, where

$$
e_{m+n}=(1,1, \ldots, 1) \in \mathbb{R}^{m+n}, \quad m=2 p^{2}, n=p^{2}+2 .
$$

We choose the preconditioning matrix $Q=\operatorname{diag}\left(B^{T} A^{-1} B\right)$. All computations for the GSOR, MSSOR, GSSOR, GMSSOR, GASOR, and MASOR methods are started from initial vector $\mathbf{x}^{(0)}=\left(x^{(0) T}, y^{(0) T}\right)^{T}=(0,0, \ldots, 0)^{T}$ and the iteration is terminated once the current iterate $\mathbf{x}^{(k)}$ satisfies

$$
\begin{aligned}
\mathrm{RES} & =\frac{\sqrt{\left\|f-A x^{(k)}-B y^{(k)}\right\|_{2}^{2}+\left\|g-B^{*} x^{(k)}\right\|_{2}^{2}}}{\sqrt{\|f\|_{2}^{2}+\|g\|_{2}^{2}}} \\
& <10^{-6}
\end{aligned}
$$

or the maximum prescribed number of iterations $k_{\max }=10 p$ is exceeded.

In Table 4, for various problem sizes $p$, we list the theoretical optimal iteration parameters of the iteration matrices 
TABLE 4: Parameters for the six methods and the corresponding pseudospectral radii.

\begin{tabular}{|c|c|c|c|c|}
\hline Method & $p$ & 16 & 24 & 32 \\
\hline \multirow{3}{*}{ GSOR } & $\omega^{*}$ & 0.7896 & 0.7398 & 0.6984 \\
\hline & $\tau^{*}$ & 1.1050 & 1.2442 & 1.3671 \\
\hline & $\rho^{*}$ & 0.4651 & 0.5650 & 0.5583 \\
\hline \multirow{2}{*}{ MSSOR } & $\omega^{*}$ & 0.45 & 0.4817 & 0.47 \\
\hline & $\rho^{*}$ & 0.5500 & 0.5684 & 0.6793 \\
\hline \multirow{3}{*}{ GSSOR } & $\omega^{*}$ & 1.4587 & 1.5110 & 1.5492 \\
\hline & $\tau^{*}$ & 0.4100 & 0.4444 & 0.4722 \\
\hline & $\rho^{*}$ & 0.4651 & 0.5650 & 0.5583 \\
\hline \multirow{3}{*}{ GMSSOR } & $\omega_{\exp }$ & 1.4600 & 1.5000 & 1.5400 \\
\hline & $\tau_{\exp }$ & 0.4300 & 0.4500 & 0.4700 \\
\hline & $\rho_{\exp }$ & 0.4876 & 0.6206 & 0.6731 \\
\hline \multirow{4}{*}{ GASOR } & $\alpha$ & 0.2300 & 0.2840 & 0.3230 \\
\hline & $\omega$ & 0.7700 & 0.7160 & 0.6770 \\
\hline & $\tau$ & 0.7097 & 0.7654 & 0.8095 \\
\hline & $\rho$ & 0.4796 & 0.5329 & 0.5683 \\
\hline \multirow{4}{*}{ MASOR } & $\omega$ & 0.7700 & 0.7160 & 0.6770 \\
\hline & $\alpha$ & 0.2300 & 0.2840 & 0.3230 \\
\hline & $\beta$ & 0.3896 & 0.5902 & 0.7418 \\
\hline & $\rho$ & 0.4796 & 0.5329 & 0.5683 \\
\hline
\end{tabular}

of the GSOR, MSSOR, GSSOR, GMSSOR, GASOR, and MASOR methods for solving singular saddle point problem. For the GSOR, MSSOR, and GSSOR methods, we take their optimal parameters as in $[5,6,49]$. For the GMSSOR method, the parameters are chosen as [42]. Furthermore, we take the parameters of the GASOR and MASOR methods which result in the least numbers of iterations for this numerical example. As mentioned in Example 1, we only choose them by trial and error. Furthermore, the pseudospectral radii of the iteration matrices of these methods are also presented in this table.

Comparing the results in Table 4, we observe that the pseudospectral radii of the GASOR and MASOR methods are smaller than those of the MSSOR and GMSSOR methods. It also can be seen that the pseudospectral radii of the GASOR and MASOR methods for Example 2 are almost the same as the ones of the GSOR and GSSOR methods. However, the GASOR and MASOR methods outperform the GSOR and GSSOR methods in terms of less CPU times and iteration steps. In Table 5, we present the iteration numbers (IT), CPU times (CPU), and relative residual (RES) of the testing iteration methods with different problem sizes $p$. In this table, we use the bold numbers to indicate the smallest and the second smallest CPU times and iteration numbers in each column. Dates presented in Table 5 reveal that the GSOR, MSSOR, GSSOR, GMSSOR, GASOR, and MASOR methods succeed in producing high-quality approximate solutions in all cases. The iteration numbers and CPU times of all methods improve with size growing. Moreover, the GASOR and MASOR methods both use the least iteration numbers and CPU times compared with the GSOR, MSSOR, GSSOR,
TABLE 5: Numerical results for the six methods.

\begin{tabular}{lcccc}
\hline Method & $p$ & 16 & 24 & 32 \\
\hline \multirow{4}{*}{ GSOR } & IT & 21 & 25 & 27 \\
& CPU & 0.2679 & 0.6841 & 1.6852 \\
& RES & $2.44 e-07$ & $5.65 e-07$ & $9.02 e-07$ \\
\hline \multirow{3}{*}{ MSSOR } & IT & 25 & 25 & 32 \\
& CPU & 0.3586 & 0.9891 & 2.9142 \\
& RES & $5.21 e-07$ & $6.06 e-07$ & $9.43 e-07$ \\
\hline \multirow{4}{*}{ GSSOR } & IT & 22 & 25 & 28 \\
& CPU & 0.3404 & 0.9095 & 2.5249 \\
& RES & $6.13 e-07$ & $7.40 e-07$ & $8.19 e-07$ \\
\hline \multirow{5}{*}{ GMSSOR } & IT & 21 & 27 & 31 \\
& CPU & 0.4154 & 1.0380 & 2.6683 \\
& RES & $7.57 e-07$ & $7.32 e-07$ & $9.57 e-07$ \\
\hline \multirow{5}{*}{ GASOR } & IT & $\mathbf{2 0}$ & $\mathbf{2 3}$ & $\mathbf{2 6}$ \\
& CPU & $\mathbf{0 . 2 5 8 2}$ & $\mathbf{0 . 6 2 7 3}$ & $\mathbf{1 . 6 3 6 7}$ \\
& RES & $5.98 e-07$ & $8.20 e-07$ & $4.95 e-07$ \\
\hline \multirow{3}{*}{ MASOR } & IT & $\mathbf{2 0}$ & $\mathbf{2 3}$ & $\mathbf{2 6}$ \\
& CPU & $\mathbf{0 . 2 6 1 5}$ & $\mathbf{0 . 6 8 0 2}$ & $\mathbf{1 . 6 1 9 4}$ \\
& RES & $5.98 e-07$ & $8.20 e-07$ & $4.95 e-07$ \\
\hline
\end{tabular}

and GMSSOR methods. Furthermore, we can observe that the performance of the GASOR and MASOR methods is the same. It can be seen that the performance of the GASOR and MASOR methods is much better than other methods, but the parameters of these two methods are not the optimal parameters, and other methods take optimal or experimentally found optimal parameters. Hence, it is anticipated that the GASOR and MASOR methods with the optimal parameters would be much better than the other four methods.

In Figure 4, the graphs of RES(log 10) against number of iterations in Table 5 for four different sizes are derived. It clearly shows that the six methods are semiconvergent while the GASOR and MASOR methods converge faster. However, the parameters of the GASOR and MASOR methods are not optimal and only lie in the convergence regions of these two methods.

\section{Conclusions}

In this paper, we propose two new methods called GASOR and MASOR methods, respectively, and study the convergence and semiconvergence of these two new methods for solving nonsingular and singular saddle point problems, respectively. Numerical results given in Section 5 (Tables 15 and Figures 1-4) show that the convergence rates of the GASOR and MASOR methods are better than the SOR-like, GSOR, MSOR-like, GSSOR, MSSOR, GMSSOR, and ASOR methods even though they are implemented with the optimal or the experimentally found optimal parameters. Numerical results present the feasibility and effectiveness of the GASOR and MASOR methods for solving both nonsingular and singular saddle point problems.

Since the optimal parameters were not used for the GASOR and MASOR methods in the numerical experiments, 

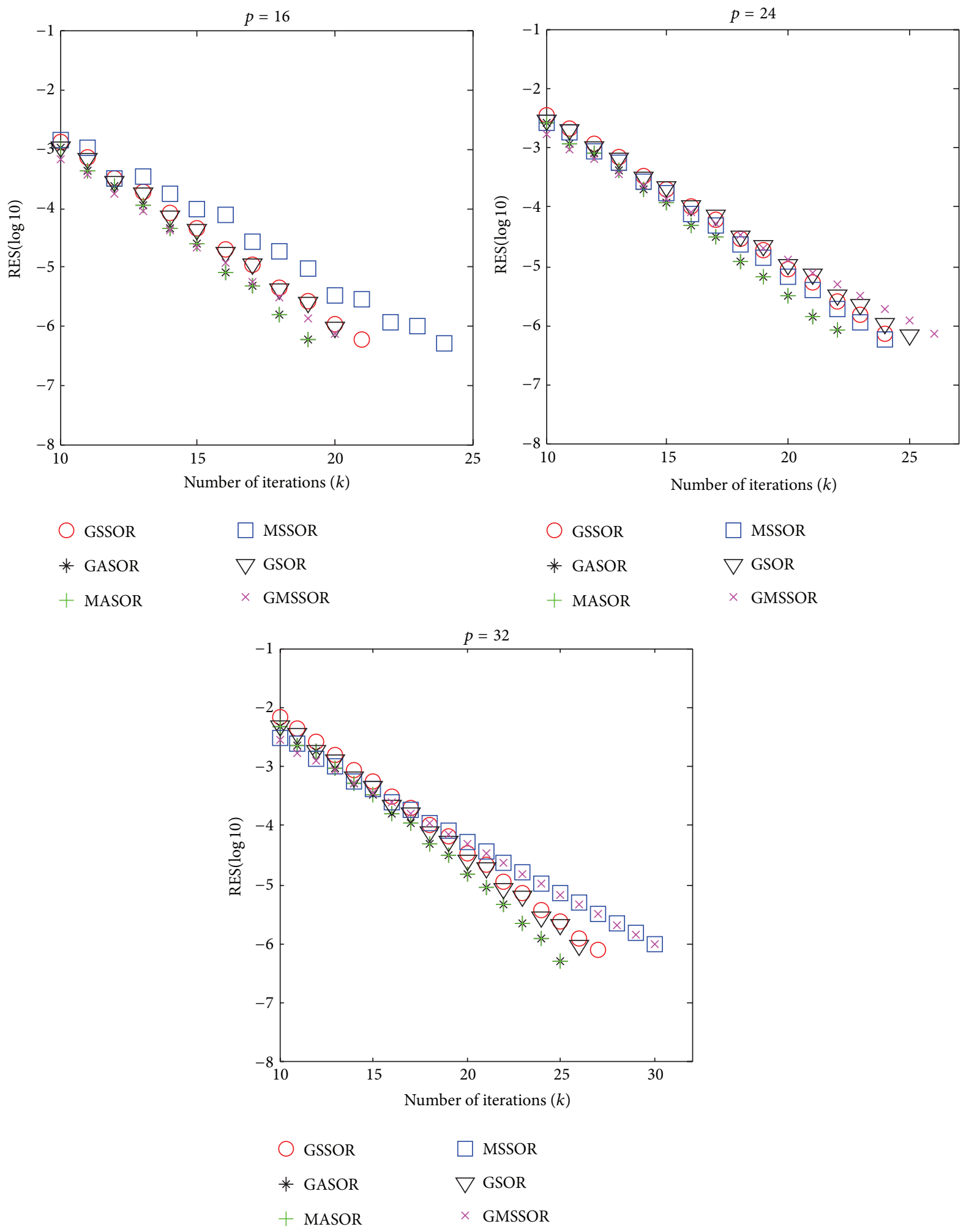

FIGURE 4: The iteration curves of algorithms for $p=16, p=24$, and $p=32$.

it is anticipated that the GASOR and MASOR methods with the optimal parameters would be much better than the other methods. Thus, it would be nice if we can find the optimal parameters for which the convergence rates of the GASOR and MASOR methods are best. Future work will include numerical or theoretical studies for finding the optimal values of $\alpha, \omega$, and $\tau$ for the GASOR method and the optimal values of $\alpha, \beta$, and $\omega$ for the MASOR method. 


\section{Competing Interests}

The authors declare that they have no competing interests.

\section{Acknowledgments}

The authors would like to express their sincere gratitude to the anonymous reviewers for their valuable comments and suggestions, which actually stimulated the presentation of this paper. This work is supported by the National Natural Science Foundations of China (no. 11171273) and sponsored by Innovation Foundation for Doctor Dissertation of Northwestern Polytechnical University (no. CX201628).

\section{References}

[1] M. Benzi, G. H. Golub, and J. Liesen, "Numerical solution of saddle point problems," Acta Numerica, vol. 14, pp. 1-137, 2005.

[2] Z.-Z. Bai, G. H. Golub, and J.-Y. Pan, "Preconditioned HERmitian and skew-HERmitian splitting methods for non-HERmitian positive semidefinite linear systems," Numerische Mathematik, vol. 98, no. 1, pp. 1-32, 2004.

[3] F. Brezzi and M. Fortin, Mixed and Hybrid Finite Element Methods, Springer, New York, NY, USA, 1991.

[4] J.-Y. Yuan, "Numerical methods for generalized least squares problems," Journal of Computational and Applied Mathematics, vol. 66, no. 1-2, pp. 571-584, 1996.

[5] Z.-Z. Bai, B. N. Parlett, and Z.-Q. Wang, "On generalized successive overrelaxation methods for augmented linear systems," Numerische Mathematik, vol. 102, no. 1, pp. 1-38, 2005.

[6] Z. Chao, N.-M. Zhang, and Y.-Z. Lu, "Optimal parameters of the generalized symmetric SOR method for augmented systems," Journal of Computational and Applied Mathematics, vol. 266, pp. 52-60, 2014.

[7] M. T. Darvishi and P. Hessari, "Symmetric SOR method for augmented systems," Applied Mathematics and Computation, vol. 183, no. 1, pp. 409-415, 2006.

[8] G. H. Golub, X. Wu, and J.-Y. Yuan, "SOR-like methods for augmented systems," BIT Numerical Mathematics, vol. 41, no. 1, pp. 71-85, 2001.

[9] P. Guo, C.-X. Li, and S.-L. Wu, "A modified SOR-like method for the augmented systems," Journal of Computational and Applied Mathematics, vol. 274, pp. 58-69, 2015.

[10] J.-C. Li and X. Kong, "Optimal parameters of GSOR-like methods for solving the augmented linear systems," Applied Mathematics and Computation, vol. 204, no. 1, pp. 150-161, 2008.

[11] X.-H. Shao, Z. Li, and C.-J. Li, "Modified SOR-like method for the augmented system," International Journal of Computer Mathematics, vol. 84, no. 11, pp. 1653-1662, 2007.

[12] Z.-Z. Bai and Z.-Q. Wang, "On parameterized inexact Uzawa methods for generalized saddle point problems," Linear Algebra and Its Applications, vol. 428, no. 11-12, pp. 2900-2932, 2008.

[13] J. H. Bramble, J. E. Pasciak, and A. T. Vassilev, "Analysis of the inexact Uzawa algorithm for saddle point problems," SIAM Journal on Numerical Analysis, vol. 34, no. 3, pp. 1072-1092, 1997.

[14] Y. Cao, M.-Q. Jiang, and L.-Q. Yao, "New choices of preconditioning matrices for generalized inexact parameterized iterative methods," Journal of Computational and Applied Mathematics, vol. 235, no. 1, pp. 263-269, 2010.
[15] Y. Cao and S.-C. Yi, "A class of Uzawa-PSS iteration methods for nonsingular and singular non-Hermitian saddle point problems," Applied Mathematics and Computation, vol. 275, pp. 4149, 2016.

[16] H. C. Elman and G. H. Golub, "Inexact and preconditioned Uzawa algorithms for saddle point problems," SIAM Journal on Numerical Analysis, vol. 31, no. 6, pp. 1645-1661, 1994.

[17] J.-H. Yun, "Variants of the Uzawa method for saddle point problem," Computers \& Mathematics with Applications, vol. 65, no. 7, pp. 1037-1046, 2013.

[18] J.-J. Zhang and J.-J. Shang, "A class of Uzawa-SOR methods for saddle point problems," Applied Mathematics and Computation, vol. 216, no. 7, pp. 2163-2168, 2010.

[19] Z.-Z. Bai, "Optimal parameters in the HSS-like methods for saddle-point problems," Numerical Linear Algebra with Applications, vol. 16, no. 6, pp. 447-479, 2009.

[20] Z.-Z. Bai and G. H. Golub, "Accelerated Hermitian and skewHermitian splitting iteration methods for saddle-point problems," IMA Journal of Numerical Analysis, vol. 27, no. 1, pp. 1-23, 2007.

[21] Z.-Z. Bai, G. H. Golub, and C.-K. Li, "Optimal parameter in Hermitian and skew-Hermitian splitting method for certain two-by-two block matrices," SIAM Journal on Scientific Computing, vol. 28, no. 2, pp. 583-603, 2006.

[22] Z.-Z. Bai, G. H. Golub, L.-Z. Lu, and J.-F. Yin, "Block triangular and skew-Hermitian splitting methods for positive-definite linear systems," SIAM Journal on Scientific Computing, vol. 26, no. 3, pp. 844-863, 2005.

[23] Z.-Z. Bai, G. H. Golub, and M. K. Ng, "Hermitian and skewHermitian splitting methods for non-Hermitian positive definite linear systems," SIAM Journal on Matrix Analysis and Applications, vol. 24, no. 3, pp. 603-626, 2003.

[24] M.-Q. Jiang and Y. Cao, "On local Hermitian and skew-Hermitian splitting iteration methods for generalized saddle point problems," Journal of Computational and Applied Mathematics, vol. 231, no. 2, pp. 973-982, 2009.

[25] Z.-Z. Bai and G.-Q. Li, "Restrictively preconditioned conjugate gradient methods for systems of linear equations," IMA Journal of Numerical Analysis, vol. 23, no. 4, pp. 561-580, 2003.

[26] Z.-Z. Bai and Z.-Q. Wang, "Restrictive preconditioners for conjugate gradient methods for symmetric positive definite linear systems," Journal of Computational and Applied Mathematics, vol. 187, no. 2, pp. 202-226, 2006.

[27] Z.-Z. Bai, "Structured preconditioners for nonsingular matrices of block two-by-two structures," Mathematics of Computation, vol. 75, no. 254, pp. 791-815, 2006.

[28] Z.-Z. Bai, M. K. Ng, and Z.-Q. Wang, "Constraint preconditioners for symmetric indefinite matrices," SIAM Journal on Matrix Analysis and Applications, vol. 31, no. 2, pp. 410-433, 2009.

[29] J.-Y. Pan, M. K. Ng, and Z.-Z. Bai, "New preconditioners for saddle point problems," Applied Mathematics and Computation, vol. 172, no. 2, pp. 762-771, 2006.

[30] X. Li, Y.-J. Wu, A.-L. Yang, and J.-Y. Yuan, "Modified accelerated parameterized inexact Uzawa method for singular and nonsingular saddle point problems," Applied Mathematics and Computation, vol. 244, pp. 552-560, 2014.

[31] Z.-Z. Liang and G.-F. Zhang, "On semi-convergence of a class of Uzawa methods for singular saddle-point problems," Applied Mathematics and Computation, vol. 247, pp. 397-409, 2014.

[32] G.-F. Zhang and S.-S. Wang, "A generalization of parameterized inexact Uzawa method for singular saddle point problems," 
Applied Mathematics and Computation, vol. 219, no. 9, pp. 42254231, 2013.

[33] N.-M. Zhang, T.-T. Lu, and Y.-M. Wei, "Semi-convergence analysis of Uzawa methods for singular saddle point problems," Journal of Computational and Applied Mathematics, vol. 255, pp. 334-345, 2014.

[34] B. Zheng, Z.-Z. Bai, and X. Yang, "On semi-convergence of parameterized Uzawa methods for singular saddle point problems," Linear Algebra and Its Applications, vol. 431, no. 5-7, pp. 808-817, 2009.

[35] P. N. Njeru and X.-P. Guo, "Accelerated SOR-like methodfor augmented linear systems," BIT Numerical Mathematics, 2015.

[36] D. M. Young, Iterative Solution of Large Linear Systems, Academic Press, New York, NY, USA, 1971.

[37] S.-S. Wang and G.-F. Zhang, "Preconditioned AHSS iteration method for singular saddle point problems," Numerical Algorithms, vol. 63, no. 3, pp. 521-535, 2013.

[38] A.-L. Yang and Y.-J. Wu, "The Uzawa-HSS method for saddlepoint problems," Applied Mathematics Letters, vol. 38, pp. 38-42, 2014.

[39] A.-L. Yang, X. Li, and Y.-J. Wu, "On semi-convergence of the Uzawa-HSS method for singular saddle-point problems," Applied Mathematics and Computation, vol. 252, pp. 88-98, 2015.

[40] S.-X. Miao and Y. Cao, "Semi-convergence of the generalized local HSS method for singular saddle point problems," Revista de la Unión Matemática Argentina, vol. 55, no. 2, pp. 71-80, 2014.

[41] M.-Z. Zhu, "A generalization of the local Hermitian and skewHermitian splitting iteration methods for the non-Hermitian saddle point problems," Applied Mathematics and Computation, vol. 218, no. 17, pp. 8816-8824, 2012.

[42] L.-J. Zhou and N.-M. Zhang, "Semi-convergence analysis of GMSSOR methods for singular saddle point problems," Computers \& Mathematics with Applications, vol. 68, no. 5, pp. 596605, 2014.

[43] L.-T. Zhang, T.-Z. Huang, S.-H. Cheng, and Y.-P. Wang, "Convergence of a generalized MSSOR method for augmented systems," Journal of Computational and Applied Mathematics, vol. 236, no. 7, pp. 1841-1850, 2012.

[44] C.-R. Chen and C.-F. Ma, "A generalized shift-splitting preconditioner for saddle point problems," Applied Mathematics Letters, vol. 43, pp. 49-55, 2015.

[45] C.-R. Chen and C.-F. Ma, "A generalized shift-splitting preconditioner for singular saddle point problems," Applied Mathematics and Computation, vol. 269, pp. 947-955, 2015.

[46] H.-T. Fan and B. Zheng, "A preconditioned GLHSS iteration method for non-Hermitian singular saddle point problems," Computers \& Mathematics with Applications, vol. 67, no. 3, pp. 614-626, 2014.

[47] G.-F. Zhang, L.-D. Liao, and Z.-Z. Liang, "On parameterized generalized skew-Hermitian triangular splitting iteration method for singular and nonsingular saddle point problems," Applied Mathematics and Computation, vol. 254, pp. 340-359, 2015.

[48] G.-F. Zhang and Q.-H. Lu, "On generalized symmetric SOR method for augmented systems," Journal of Computational and Applied Mathematics, vol. 219, no. 1, pp. 51-58, 2008.

[49] S.-L. Wu, T.-Z. Huang, and X.-L. Zhao, "A modified SSOR iterative method for augmented systems," Journal of Computational and Applied Mathematics, vol. 228, no. 1, pp. 424-433, 2009.
[50] Z.-Z. Bai, L. Wang, and J.-Y. Yuan, "Weak-convergence theory of quasi-nonnegative splittings for singular matrices," Applied Numerical Mathematics. An IMACS Journal, vol. 47, no. 2, pp. 75-89, 2003.

[51] A. Berman and R. J. Plemmons, Nonnegative Matrices in the Mathematical Sciences, Academic Press, New York, NY, USA, 1979.

[52] A. Berman and R. J. Plemmons, Nonnegative Matrices in the Mathematical Sciences, Society for Industrial and Applied Mathematics, Philadelphia, Pa, USA, 1994.

[53] X. Li, A.-L. Yang, and Y.-J. Wu, "Parameterized preconditioned HERmitian and skew-HERmitian splitting iteration method for saddle-point problems," International Journal of Computer Mathematics, vol. 91, no. 6, pp. 1224-1238, 2014.

[54] Y. Cao, J. Du, and Q. Niu, "Shift-splitting preconditioners for saddle point problems," Journal of Computational and Applied Mathematics, vol. 272, pp. 239-250, 2014.

[55] Y. Cao, M.-Q. Jiang, and Y.-L. Zheng, "A splitting preconditioner for saddle point problems," Numerical Linear Algebra with Applications, vol. 18, no. 5, pp. 875-895, 2011. 


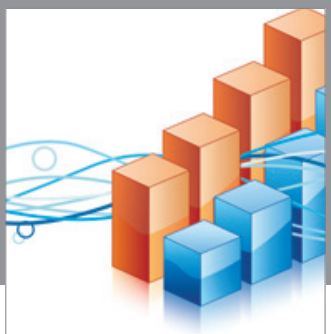

Advances in

Operations Research

vatem alat4

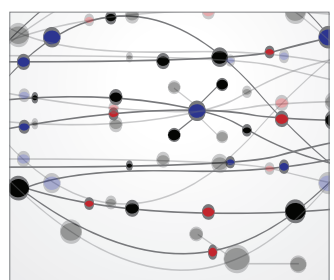

\section{The Scientific} World Journal
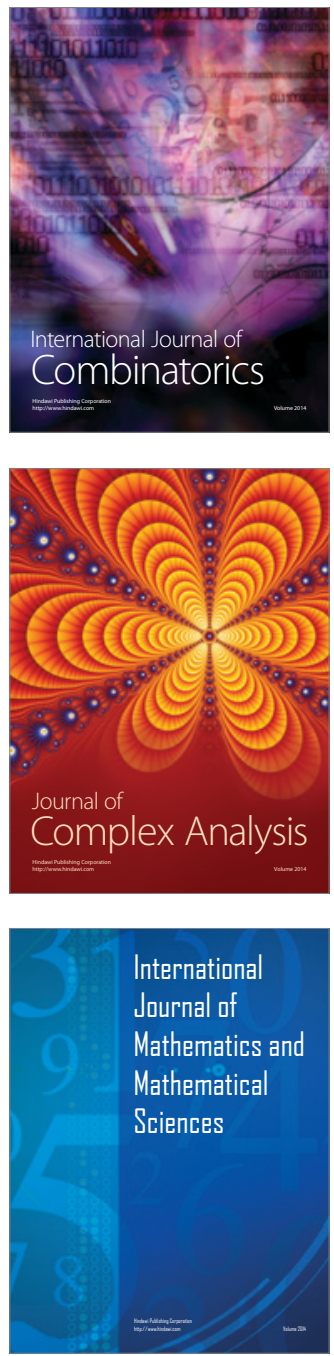
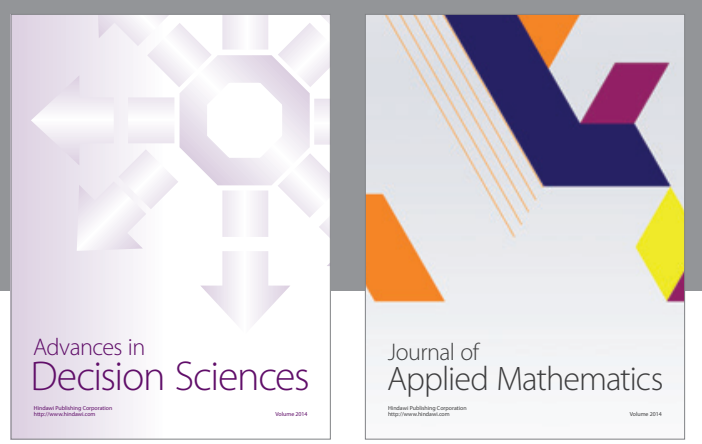

Algebra

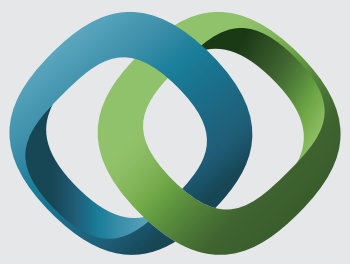

\section{Hindawi}

Submit your manuscripts at

http://www.hindawi.com
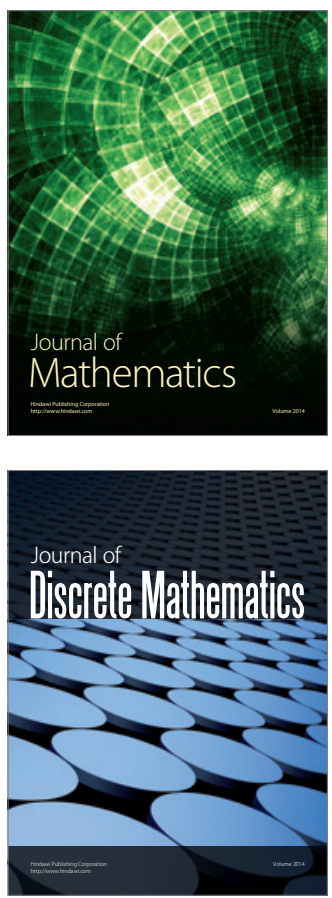

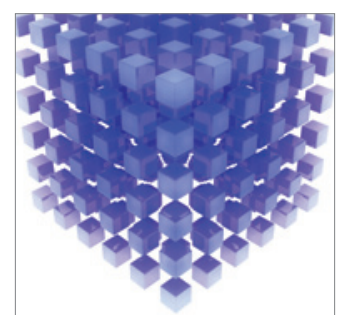

Mathematical Problems in Engineering
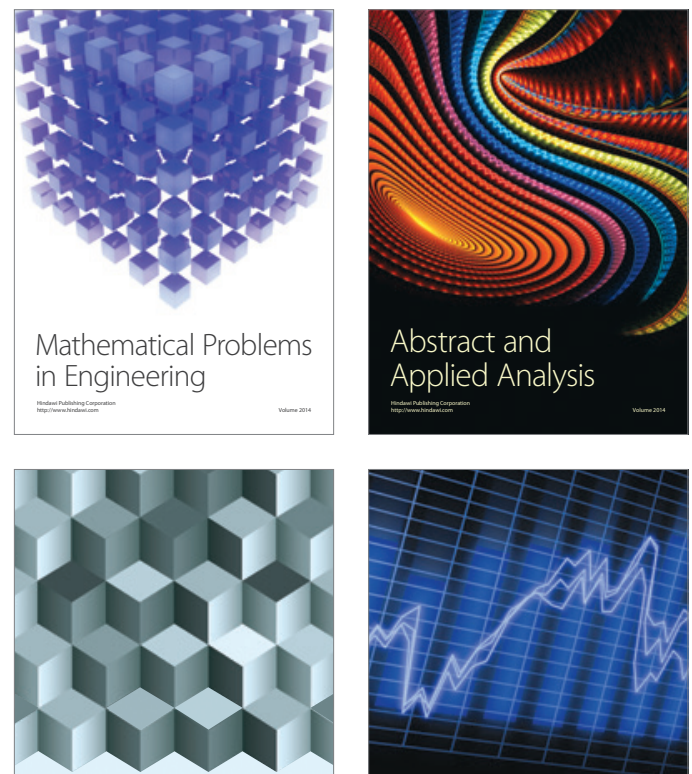

Journal of

Function Spaces

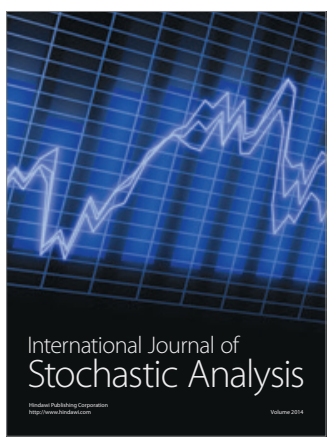

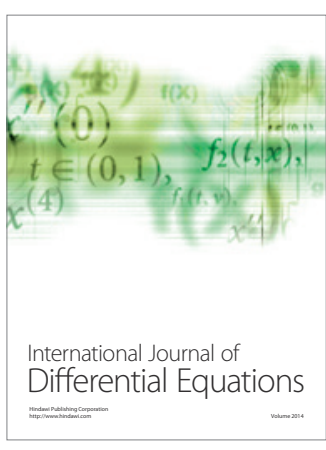
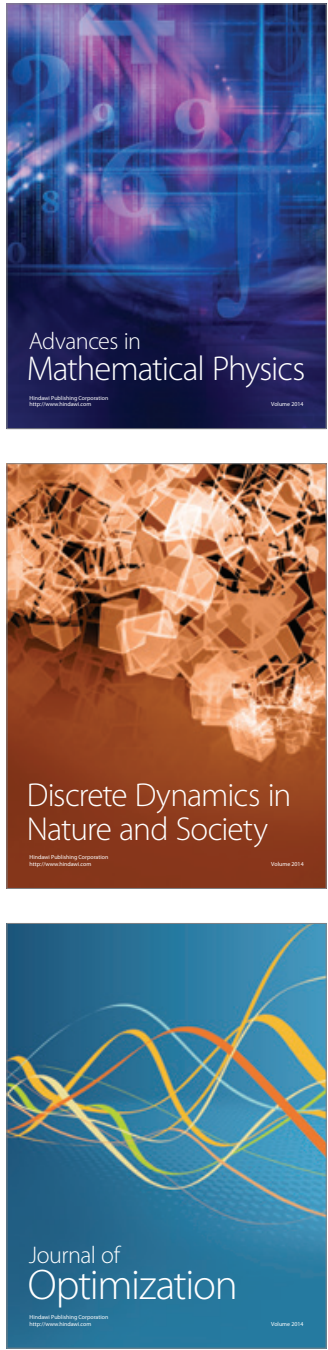